\title{
„Megatrend“ Wandern und Trekking? - Eine narrative Synopse von MarkTforschungSDaten, MEdienberichten UND ANDEREN, VERMEINTLICHEN INDIKATOREN ${ }^{1)}$
}

\author{
Luisa VogT, Soest - Birmensdorf* \\ mit 4 Abb. und 2 Tab. im Text
}

\section{INHALT}

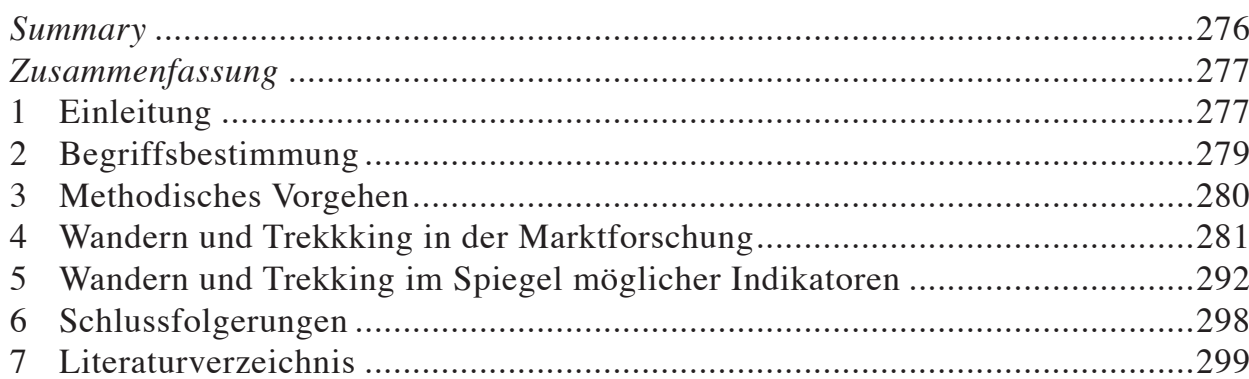

\section{Summary}

Megatrend Hiking and Trekking? - A Narrative Synopsis of Market Research Data, Media Reports and Further Pretended Indicators

In the last few years, hiking and trekking have attracted large media attention as modern trend sports. Furthermore, in the few scientific publications on this market segment, a high significance has been assigned to hiking. The widely consistent media opinion has been adopted in academia without any examination. The assumption of the hiking trend has not yet been reviewed, despite the financial support by regional and tourism politics for the development of hiking tourism destinations in Germany and

\footnotetext{
1) Fabian Waltert, Daniel Baumgartner und anonymen Gutachtern sei für Anregungen herzlich gedankt.

* Dr. phil. Dipl.-Geogr. Luisa Vogt, Institut für Green Technology \& Ländliche Entwicklung, FH Südwestfalen, Lübecker Ring, D-59494 Soest; E-mail: vogt@fh-swf.de, http://www3.fh-swf.de/igreen/ und Eidg. Forschungsanstalt für Wald, Schnee und Landschaft WSL, Forschungseinheit Wirtschaftsund Sozialwissenschaften, Forschungsgruppe Regionalökonomie und -entwicklung, Zürcherstraße 111, CH-8903 Birmensdorf, http://www.wsl.ch
} 
in the Alps. Based on German market research data, this article shows that hiking is indeed an important leisure and holiday activity. However, its popularity is declining rather than growing. The study also contains a narrative synopsis of media reports and developments of markets that are related to hiking and trekking. The developments found there were the opposite of the "real" hiking and trekking developments. In connection with the repositioning of stagnating traditional destinations, hiking and trekking attract growing attention. The paper closes with recommendations for tourism service providers and some methodological conclusions on handling secondary data and indicators.

\section{Zusammenfassung}

Wandern und Trekking haben in den letzten Jahren eine große Medienresonanz als Trendsport erfahren. Auch in den wenigen wissenschaftlichen Publikationen über dieses Marktsegment wird dem Wandern bei einer ungeprüften Übernahme der weitgehend konsistenten Medienmeinung ein großer, steigender Stellenwert bei den Freizeit- und Urlaubsaktivitäten beigemessen. Die These über den Wandertrend wurde bislang jedoch nie einer Prüfung unterzogen, obwohl mit regional- und tourismuspolitischer Unterstützung in Deutschland und in den Alpen wandertouristische Destinationen entwickelt werden. Der vorliegende Beitrag zeigt anhand von deutschen Marktforschungsdaten, dass das Wandern als Aktivität in der Freizeit und im Urlaub häufig ausgeübt wird, aber an Popularität eher einbüßt als dazu gewinnt. Gegenläufig dazu erfahren Wandern und Trekking in den Medien und auf den Wandern und Trekking verwandten Märkten eine steigende Beachtung. In einer qualitativen Analyse wird diese Entwicklung aufgezeigt und in den Zusammenhang der Neupositionierung stagnierender und traditioneller Destinationen gestellt. Der Beitrag schließt mit inhaltlichen Schlussfolgerungen für die touristische Angebotsseite und mit methodischen Quintessenzen für den Umgang mit Sekundärstatistiken und Indikatoren.

\section{Einleitung}

Wandern und Trekking gelten als sehr beliebte außerhäusliche Freizeitaktivitäten (Spiess et al. 2008). ThienE und SCARPa (2008, S. 263f.) begründen dies für die Alpen logisch-deduktiv mit niedrigen Eintrittsbarrieren: "[I]t is cheap, does not require complicated skills and can be performed at a variety of levels across the dense network of hiking tracks that criss-cross the alpine territory". Nicht nur diese Autoren nehmen einen hohen Stellenwert des Wanderns und Trekkings als (tages-)touristische Marktsegmente an. Eine Popularität lässt auch die große Medienpräsenz des Wanderns und Trekkings vermuten. So berichteten in den letzten fünf Jahren praktisch alle deutschen Medien über diese Freizeitaktivität als Trend (vgl. Prantl 2007). Auf die Annahme eines großen Marktpotenzials stützen sich auch Regionalpolitiker, die den Wandertourismus ,als Schlüsselelement in der lokalen Entwicklung“ erachten (Europäısche 
Beobachtungsstelle Leader 2001, S. 5) und eine Angebotsentwicklung entsprechend fördern. Die Europäische Union und die Alpenländer investierten z.B. von 2001 bis 2007 fünf Millionen Euro für die Lancierung der Via Alpina, eines alpenweiten Trekkingwegenetzes (VIA Alpina 2008). Potenzial für die Entwicklung ländlicher Regionen erkennen auch die Arbeitskreise „Wirtschaftsförderung“ sowie „Freizeit und Tourismus" des Deutschen Verbandes für Angewandte Geographie (DVAG), die im September 2009 eine Tagung zum „Megatrend Wandern“ ausrichteten.

Die proklamierte Beliebtheit und - dem Trendbegriff ${ }^{2}$ innewohnende - wachsende Popularität des Wanderns und Trekkings als Freizeit- und Urlaubsaktivität findet sich nicht in einer entsprechenden wissenschaftlichen Aufarbeitung wieder. Der Autorin sind keine Studien bekannt, die sich mit der Entwicklung dieses Marktsegments befassen oder die z.B. mögliche Einflussfaktoren hierfür untersuchen. Eine kritische Überprüfung, inwiefern sich Wandern und Trekking tatsächlich einer großen und wachsenden Nachfrage erfreuen, erscheint jedoch unter anderem wegen gegenläufiger Meldungen angebracht. So musste zum Beispiel der französische Alpenverein in seinen Hütten zwischen 2003 und 2005 einen Übernachtungsrückgang um 10\% feststellen (ALPMEDIA News 2006). Zudem sind die Ergebnisse von Marktforschungsinstituten nicht immer widerspruchsfrei. In einer repräsentativen Erhebung der deutschen Bevölkerung über 14 Jahren nennen z.B. gerade 25\% das Wandern als eine von ihnen ausgeübte Sportaktivität (STATISTA 2008c). In einer weiteren Erhebung geben aber 56\% an zu wandern (IFD 2007), wobei in den Medien fast ausschließlich Zahlen wie die letztere zitiert werden (z.B. BRUNNER 2004). Diskrepante Resultate finden sich auch zur Entwicklung des Wanderns in den letzten zwei Jahrzehnten.

Klassischerweise beschäftigt sich die Tourismusgeographie mit Fragestellungen in touristischen Zielgebieten. Untersuchungen der Nachfrage mit Fokus auf das Quellgebiet sind jedoch unerlässlich, wenn z.B. Einflussfaktoren für die Wettbewerbsfähigkeit von Destinationen analysiert werden.

Am Wettbewerbsfaktor der touristischen Nachfrage setzt der vorliegende Beitrag an. Sein Ziel ist es, den Stellenwert des Wanderns und Trekkings als Freizeit- und Urlaubsaktivität kritisch zu analysieren. Die Prüfung der Hypothese eines Trends oder gar „Megatrends“ Wandern soll einerseits gestützt auf quantitative Nachfrageanalysen erfolgen. Dabei interessiert auch ein Vergleich mit der Bedeutung und der Entwicklung anderer Freizeit- und Urlaubsaktivitäten. Andererseits sollen als weitere - teilweise auch hinsichtlich der Indikata zu diskutierende - Indikatoren „, weiche“ Kriterien herangezogen werden: die Medienpräsenz, die Themenschwerpunkte von Messen und Tagungen, der mit dem Tourismus verwandte Markt des Sportfachhandels sowie das touristische Marketing deutscher und österreichischer Destinationen.

Die Analyse richtet den Fokus bei der nachfrageseitigen Quellgebietsanalyse auf Deutschland. Deutschland ist eines der wichtigsten Entsenderländer im weltweiten

\footnotetext{
2) Als Trend lässt sich eine Veränderung oder Strömung in der Gesellschaft in Bezug auf Werte und Einstellungen, Verhalten und Handeln bezeichnen (PiLlKahn 2007). Er enthält Aussagen zur bisherigen Entwicklung und zur zukünftigen, wobei für dessen Prognose - z.B. über die Delphi-Methode - meist ceteris-paribus-Annahmen und ein linearer Verlauf zu Grunde gelegt werden. Für besonders revolutionäre und lang andauernde Trends hat sich der Begriff „Megatrend“ etabliert. Da Aussagen, die Wissen über die Zukunft versprechen, populär sind, werden oft unseriös ermittelte Trends ausgerufen.
} 
Tourismus (WTO 2007) und das wichtigste Quellgebiet des Alpentourismus; damit haben Entwicklungen auf dem deutschen Markt auch Relevanz für andere Länder und insbesondere für die Alpen und Österreich. Die Untersuchung der Angebotsseite erfolgt für Deutschland und Österreich.

Der Artikel beginnt mit einer Begriffsdifferenzierung im Wortfeld Wandern. Ein Abschnitt widmet sich dem methodischen Vorgehen des Beitrags, bevor das Marktpotenzial des Wanderns überprüft wird. Der Analyse quantitativer Aspekte des Wanderund Trekkingmarkts schließt sich die Untersuchung qualitativer Gesichtspunkte an. Der Beitrag endet mit Schlussfolgerungen für die touristische Angebotsseite und für die Tourismusforschung.

\section{Begriffsbestimmung}

Das Wortfeld zu Wandern ist groß, wobei unterschiedliche semantische Deutungen von Begriffen häufig zu Verwirrungen und Fehlinterpretationen führen. Sprachwissenschaftliche Analysen zum Begriff des Wanderns liegen leider nicht vor. Sehr allgemein lässt sich Wandern als Form der Standortveränderung definieren. Wandern bleibt auch bei einer Eingrenzung als Freizeitaktivität noch ein Sammelbegriff. So subsumiert die BRockhaus-EnZYKLOPÄDIE (2007) ,vielfältige Formen der aktiven Erholung zu Fuß, per Fahrrad, mit Boot, Skiern oder Pferd“ unter „Wandern“. Andere Wörterbücher grenzen „Wandern“ auf die Fortbewegung per pedes ein (WAHRIG-BuRfeind 2002, S. 1373). Im Sprachgebrauch dürfte dies auch die primäre Assoziation sein. Eine weitergehende Abgrenzung gegenüber anderen Formen der Freizeitmobilität zu Fuß kann über den Bewegungsablauf, die Fortbewegungsmittel, den Energieaufwand und über zeitlich-räumliche Kriterien erfolgen.

Es lässt sich ein relativ breiter Konsens von (Tages-)Touristen, Touristikern (Anbietern, Mittlern, Marktforschern) und Sportmedizinern darüber annehmen, dass der Bewegungsablauf des Wanderns dem des Gehens gleich ist. Damit bleibt in jeder Phase des Bewegungszyklus beim Wandern der Kontakt des Körpers zum Boden über die Füße bestehen. Es findet im Gegensatz zum Laufen und Joggen keine Flugphase statt. Eine hohe Zustimmung dürfte auch die Einschränkung auf die Füße als alleinige Fortbewegungsmittel finden. Aktivitäten mit technischen Hilfsmitteln (z.B. Schneeschuhe) und dem Einsatz von Händen und Armen zur Fortbewegung wären damit vom Wandern im engeren Sinn ausgeschlossen. Bergsteigen (gelegentliche Zuhilfenahme von Händen), Klettern (stetiger Einsatz von Armen und Händen sowie einer speziellen Ausrüstung) oder Nordic Walking (Nutzung von Armen und Stöcken) gehören demnach nicht zum Wandern. Bergwandern dürfte dagegen allgemein als Unterform des Wanderns aufgefasst werden.

Eine Differenzierung über den Energieaufwand dürften einige (Tages-)Touristen und Touristiker spontan nicht vornehmen. Der Energieaufwand beim Gehen hängt grundsätzlich von der Dauer der Aktivität, von der Geschwindigkeit der Fortbewegung, von der Beschaffenheit und der Steigung des Untergrunds und schließlich von individuellen Faktoren (Körpergewicht etc.) ab (AINSwORTH 2002). Es ist aber zu 
vermuten, dass tendenziell im allgemeinen Sprachgebrauch (mit regionalen Unterschieden) parallel zum sportmedizinischen Verständnis Spaziergänge implizit mit einem geringeren Energieverbrauch konnotiert werden als Wanderungen. AINSWORTH (2002) differenziert physische Aktivitäten nach ihren metabolischen Äquivalenten, d.h. der Menge Sauerstoff bzw. Energie, die ein Körper während der physischen Aktivität verbraucht. Wanderungen sind demzufolge vergleichsweise energieintensiv, wobei AinsworTh (2002) Wanderungen (hiking) mit einer Fortbewegung querfeldein gleichsetzt und jegliches Gehen unabhängig von der Geschwindigkeit, dem Untergrund und der Steigung als walking bezeichnet. (Allgemein anerkannte deutschsprachige sportmedizinische Definitionen sind der Verfasserin nicht bekannt.)

Die zeitliche Dimension dürfte allgemein als Unterscheidungskriterium zwischen Wanderungen und Weitwanderungen verwendet werden: Wanderungen dauern bis zu einem Tag, Weitwanderungen länger als einen Tag. Diese führen zudem von Ort zu Ort; d.h. es findet keine tägliche Rückkehr an einen festen Standort statt. Häufig wird der Begriff des Trekkings synonym mit Weitwandern genutzt. Teilweise findet jedoch beim Begriff Trekking eine Konnotation mit der Überwindung größerer Höhendifferenzen statt (vgl. HAUCK 1996, S. 1). Trekking wäre dann eine Weitwanderung im (Hoch-)Gebirge - unabhängig vom Ausbau der Infrastruktur (Wege, Markierung etc.; schließlich ersetzen z.B. im Himalaja häufig Führer die in den Alpen übliche Orientierungsfunktion von markierten Wegen) und unabhängig von der Region, auch wenn z.B. BEEDiE \& Hudson (2003, S. 626) meinen: "Hill walking in 'exotic places' has been redefined as trekking".

Diese versuchsweise Differenzierung der Termini dient der Sensibilisierung für deren semantische Breite. Das Begriffsverständnis ist nicht eindeutig, sodass dieses jeweils zu prüfen ist.

\section{Methodisches Vorgehen}

Um quantitative Erkenntnisse zur Höhe und Entwicklung des Wanderns und Trekkings sowie weiterer physischer Aktivitäten in Deutschland zu überprüfen, wird eine narrative Synopse vorliegender Erhebungen von Marktforschungsinstituten durchgeführt (vgl. Collins \& Fauser 2005, Educational Research Review 2008). In Deutschland liegen repräsentative, nachfrageseitige Quellgebietsbefragungen nicht im Zuständigkeitsbereich der amtlichen Statistik. Stattdessen bieten mehrere private Institute diese Dienstleistung privatwirtschaftlich an. Die Recherche nach Marktforschungsdaten begann bei den Webauftritten der in der einschlägigen Literatur (STENGER 1998) genannten Marktforschungsinstitute, die sich auf touristische Erhebungen spezialisiert haben. Eine Google Scholar-Recherche sowie eine Recherche im Online-Archiv deutscher Tages- und Wochenzeitungen führte zu Publikationen zum Thema, in denen noch weitere Marktforschungsergebnisse, vor allem zum Wandern als Freizeitaktivität, zitiert wurden. Für die Analyse des Stellenwerts und der Entwicklung von Wandern und Trekking werden alle bevölkerungsrepräsentativen Erhebungen berücksichtigt. Um methodisches Vorgehen und Zeitreihendaten jeweils ab Beginn entsprechender 
Erhebungen zu eruieren, wurden - wenn die Informationen nicht öffentlich vorlagen - Marktforschungsinstitute via E-Mail angefragt.

Grundsätzlich stehen Marktforschungsresultate der Öffentlichkeit nur in begrenztem Maß zur Verfügung. Vor allem längere diachrone Vergleiche von Marktforschungsergebnissen der gleichen Institute gestalten sich schwierig, wenn man auf frei verfügbare Quellen angewiesen ist. Zeitreihen liegen allerdings auch von wenigen Instituten vor, da Erhebungen zum Teil auch nur episodisch oder einmalig erfolgen (vgl. AgRICOLA 2003).

Die qualitative Analyse der Bedeutung und Entwicklung von Wandern und Trekking stützt sich auf Indikatoren, die vom Deutschen Wanderverband (vgl. NEUMEYER \& Dicks 2004) und in einem Beitrag von Pröвstl (2004) herangezogen werden. Die deskriptive Untersuchung der Medienpräsenz basiert auf einer unsystematischen Beobachtung von Printmedien und einer systematischen Auswertung des OnlineArchivs zweier bundesweit verbreiteter und überregional wahrgenommener deutscher Tageszeitungen, die von Tourismusmessen auf einer systematischen Recherche nach Themenschwerpunkten der drei besucherstärksten Publikumsmessen. Die Untersuchung des Sportartikelmarkts erfolgt mittels Marktforschungsdaten, wobei logisch-deduktiv das Indikatum des Indikators diskutiert wird. Der Stellenwert des Wander- und Trekkingsegments für die Vermarktung touristischer Destinationen wird an ausgewählten Beispielen deutscher und österreichischer Reiseziele deskriptiv aufgezeigt.

\section{Wandern und Trekking in der Marktforschung}

\subsection{Wandern als Freizeitaktivität}

\subsubsection{Resultate}

Vom deutschen Institut für Demoskopie (IfD) liegen seit 1986 im Rahmen der Allensbacher Markt- und Werbeträgeranalyse (AWA) Zahlen zu Freizeitbeschäftigungen vor. 1986 wanderten 54\% der Deutschen in ihrer Freizeit (IFD 2008). Dieser Wert sinkt Anfang bis Mitte der 1990er Jahre leicht auf 51\%, um nach einem Ausreißer nach oben 1997 ab 1998 bis 2000 wieder auf 54\% und bis 2005 auf 63\% zu steigen (DGF 1999, IFD 2007, vgl. Abb. 1). Seitdem nimmt er wieder deutlich ab; 2007 und 2008 geben nur noch 56\% an zu wandern (IFD 2007, BRÄMER 2008). Über die Jahre wandern zwischen 8 und 13\% der Deutschen häufig, alle anderen ab und zu (vgl. Tab. 1).

Eine ähnlich lange Zeitreihe wie die der AWA besteht nur im Rahmen des FreizeitMonitors des BAT Freizeit-Forschungsinstituts. In den Jahren 1993 bis 2003 ging den Befragungen zufolge jeweils konstant rund ein Drittel der Deutschen ${ }^{3)}$ in der Woche vor dem Erhebungszeitpunkt spazieren oder wandern (BAT FREIZEIT-ForsChungsinstituT 2002, 2003). 2004 ändert sich die Fragestellung, gefragt wird seitdem nach der Häufigkeit von Freizeitaktivitäten (vgl. Tab. 1). Nur $10 \%$ spazieren oder wandern nie,

\footnotetext{
3) Gemeint ist mit „Deutschen“ in diesem Fall und auch bei allen weiteren Erhebungen die Wohnbevölkerung Deutschlands (vgl. Tab. 1).
} 


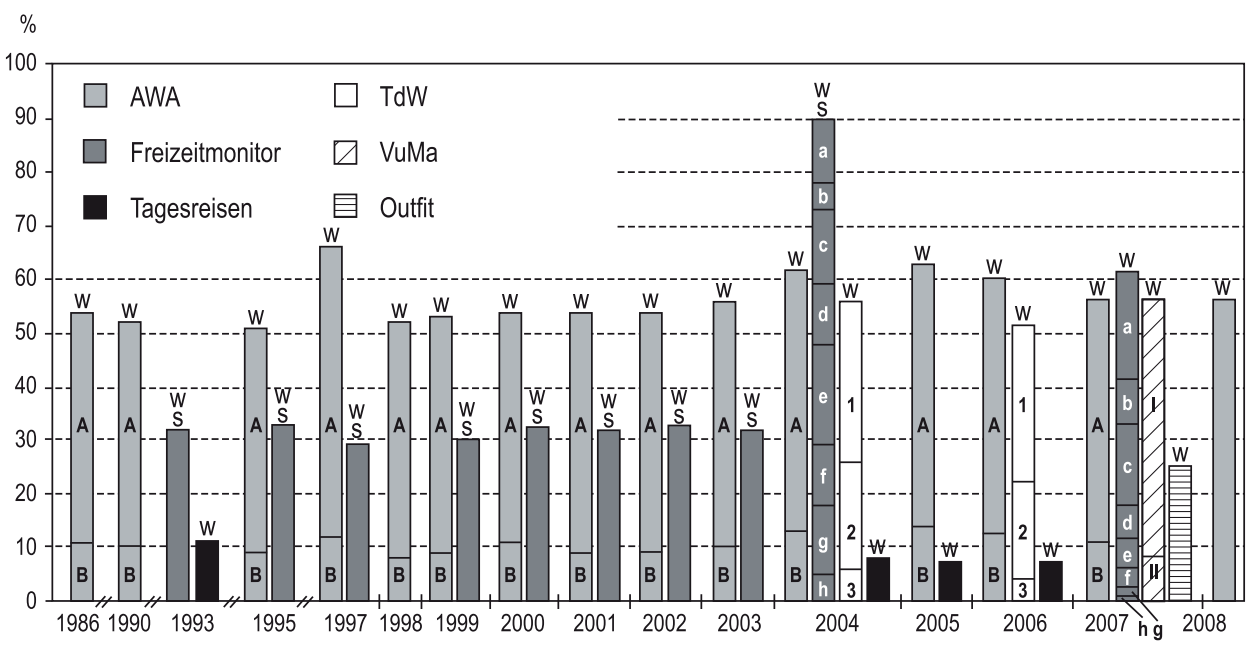

A: ab und zu, B: häufig

a: seltener als einmal jährlich, b: vielleicht einmal jährlich, c: mehrmals im Jahr, d: etwa einmal pro Monat, e: mehrmals im Monat, f: etwa einmal pro Woche, g: mehrmals in der Woche; h: täglich

1: nur selten, 2: gelegentlich, 3: regelmäßig

I: gelegentlich, II: mehrmals im Monat

w wandern

s spazieren gehen

AWA: Erhebungsbeginn 1986, Werte 1987-1994, 1996 nicht zur Verfügung gestellt; TdW, VuMa, Outfit: Erhebungsbeginn unbekannt, keine Kenntnis über fehlende Werte; Freizeitmonitor: Erhebungsbeginn 1993, keine Erhebungen 1994, 1996, 1998, 2005, 2006; Tagesreisen: bislang vier Erhebungen 1993, 2004, 2005, 2006. Vgl. zu den Fragestellungen Tab. 1.

Quellen: IfD 2007, 2007a, 2008, DGF 1999, BRÄMER 2008, Focus 2005, StATISTA 2008, 2008a-c, BAT Freizeit-Forschungsinstitut 2002-2004, Stiftung FÜr ZukunftSFragen 2008, HARrer et al. 1995, MaschKe 2005, 2007. Eigene Zusammenstellung.

Abb. 1: Entwicklung der Freizeitaktivität Wandern im Spiegel der Marktforschung

59\% hingegen einmal im Monat oder öfter (BAT FreIZEIT-Forschungsinstitut 2004). Auch der Wert für 2007 ist nicht mit den vorherigen Werten vergleichbar, denn 2007 ändert sich nochmals die Fragestellung, und es wird nun nach den Aktivitäten Wandern und Spaziergänge getrennt gefragt. 12\% der Deutschen wandern dabei mehrmals im Monat und $41 \%$ mindestens einmal im Jahr. Erst wenn auch die 20\% der Deutschen eingerechnet werden, die seltener als einmal jährlich wandern, ergibt sich ein mit der AWA in etwa kompatibler Wert (STIFTUng Für Zukunftsfragen 2008).

Die Ergebnisse der Verbrauchs- und Medienanalyse (VuMa) stimmen mit den AWAWerten weitgehend überein. Ihr zufolge wandern in den Erhebungen 2005-2007 zwar nur $9 \%$ der Deutschen mehrmals im Monat, 56\% gehen aber zumindest gelegentlich 
dem Wandern nach (Statista 2008, 2008a). ${ }^{4}$ Die Marktanalyse Typologie der Wünsche (TdW) kommt für die Jahre 2004/05 und 2006 zu ähnlichen Gesamtresultaten: Insgesamt wandern $56 \%$ bzw. $51 \%$ mindestens selten, dabei jedoch $30 \%$ bzw. später $29 \%$ „nur selten“, weitere $20 \%$ bzw. $18 \%$,gelegentlich“ und nur geringe $6 \%$ bzw. $4 \%$ „regelmäßig“ (Focus 2005, S. 5; STATista 2008b).

Auf den ersten Blick kommt die Marktanalyse Outfit für 2006/07 zu deutlich anderen Zahlen: Nur 25\% der Befragten geben Wandern als ausgeübte Sportart an (STATISTA 2008c). Letztlich entspricht dieser Wert aber in etwa dem Anteil derer, die nach der TdW gelegentlich oder regelmäßig wandern (26\% im Jahr 2004/05, 22\% 2006, s. oben).

Das Deutsche Wirtschaftswissenschaftliche Institut für Fremdenverkehr an der Universität München (dwif) analysiert das Tagesausflugsverhalten und fragt dabei nach den ausgeübten Aktivitäten. Bei der ersten Befragung 1993 wanderten (Mehrfachnennungen waren möglich) nur $11 \%$ bei ihren Ausflügen (HARRER et al. 1995). In den Befragungen 2004 bis 2006 wurde der Hauptanlass des Ausflugs erhoben: So wird bei $21 \%$ (2004), 22\% (2005) und wieder 21\% (2006) der Tagesausflüge eine ,spezielle Aktivität (z.B. Skilauf, Baden, Wandern, Surfen)“" ausgeübt (Maschke 2005, S. 81; MAschke 2007, S. 47), wobei nur $8 \%$ der Tagestouristen „wandern und bergsteigen“ (E-Mail-Mitteilung MaschKe 2008).

\subsubsection{Diskussion der Ergebnisse}

Die Bedeutung des Wanderns als Freizeitaktivität ist offensichtlich groß - in Kapitel 4.1.3 wird das Wandern noch mit anderen Freizeitaktivitäten verglichen. Auffällig sind die Differenzen bei den Erhebungsresultaten (vgl. Abb. 1). Sie haben potenziell verschiedene Gründe, wie z.B. systematische Fehler bei den Stichprobenerhebungen, methodische Divergenzen bei Hochrechnungen oder nicht erfüllte Gütekriterien (Objektivität, Reliabilität, Validität) bei den Erhebungen (vgl. STENGER 1998). Die Hauptursache für die unterschiedlichen Ergebnisse für das gleiche Jahr dürften jedoch - und dies betrifft die konkurrente Validität (RAUCHFLEISCH 1994) - die verschiedenen Fragestellungen, Frageformulierungen und die verschiedenstufigen Skalen bei den Antwortmöglichkeiten sein (vgl. auch Tab. 1): Bei der AWA umfasst die Skala drei Stufen, bei der TdW vier, bei der VuMa scheinbar drei und beim Freizeit-Monitor ab 2004 neun. Die Fragestellungen des Freizeit-Monitors bis 2003, von Outfit und die der dwif-Analyse entsprechen Ja-Nein-Fragen. Relativ ähnliche Werte generieren 2006/07 die Addition der zwei Stufen „,ab und zu“ und „häufig“ der AWA, ,gelegentlich“ und „mehrmals im Monat“ der VuMa, der drei Stufen ,selten“, „,gelegentlich“, ,,regelmäBig“ der TdW sowie der acht Stufen (von täglich bis seltener als einmal jährlich) des Freizeit-Monitors. Die Fragestellungen von Outfit und des dwif führen wohl zu einer Polarisierung und einem deutlich niedrigeren Stellenwert des Wanderns. (Zudem bleibt zwar bei Outfit die Interpretation des Begriffs „Wandern“ wie bei allen ande-

\footnotetext{
4) Leider wurden keine Informationen zur Verfügung gestellt, seit wann die Erhebungen durchgeführt werden. Zeitreihendaten liegen der Verfasserin nicht vor. Im Folgenden wird im Text nicht mehr explizit auf die Datenverfügbarkeit verwiesen (vgl. dazu generell Tab. 1, 2 und Abb. 1, 2).
} 


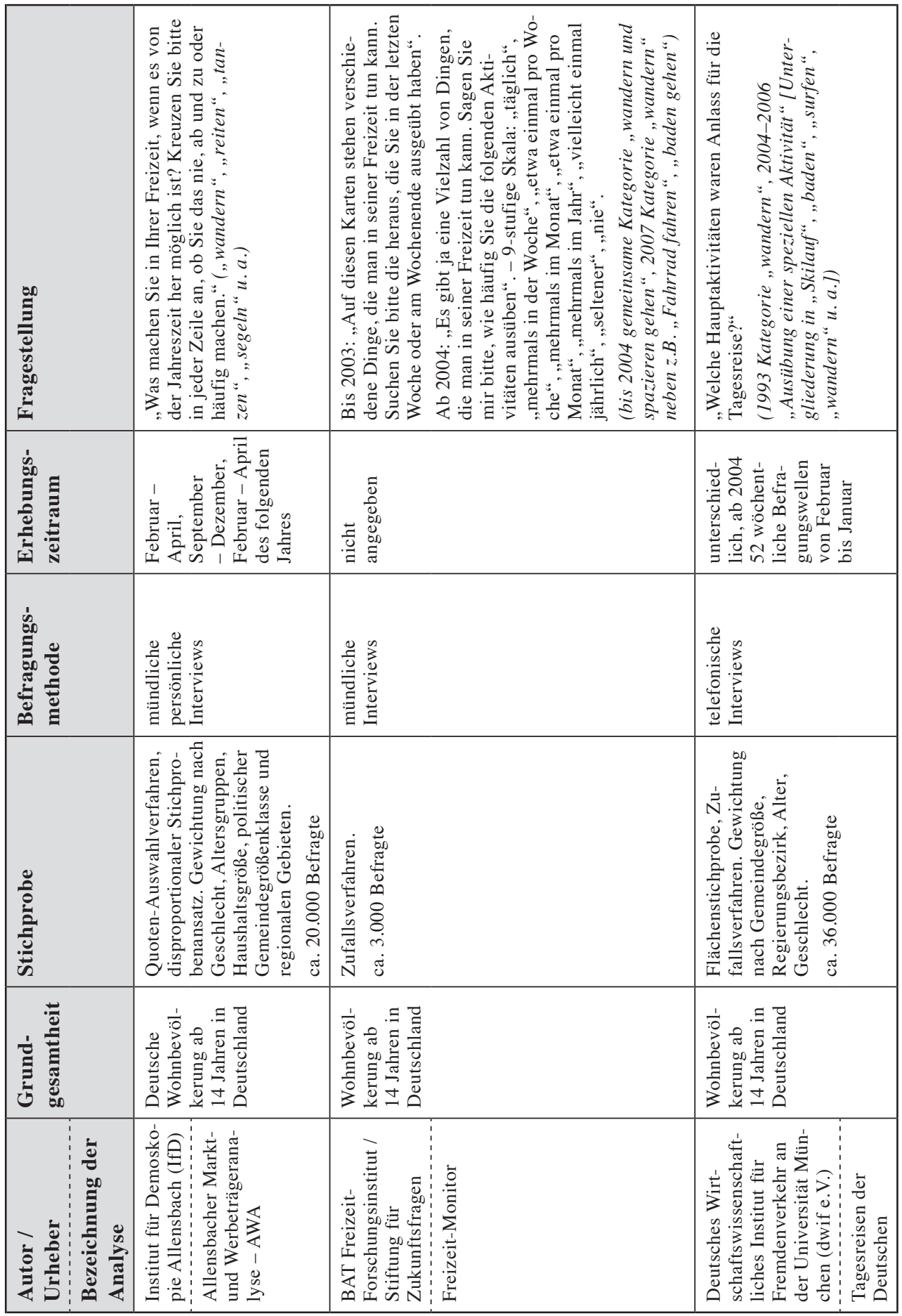


„Megatrend“ Wandern und Trekking?

\begin{tabular}{|c|c|c|c|c|c|}
\hline 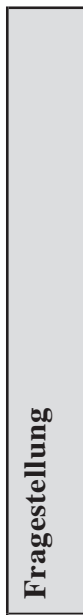 & & 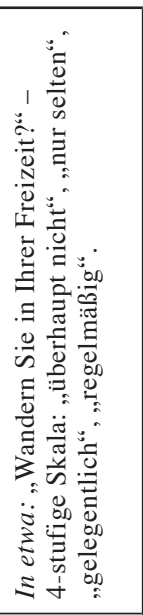 & 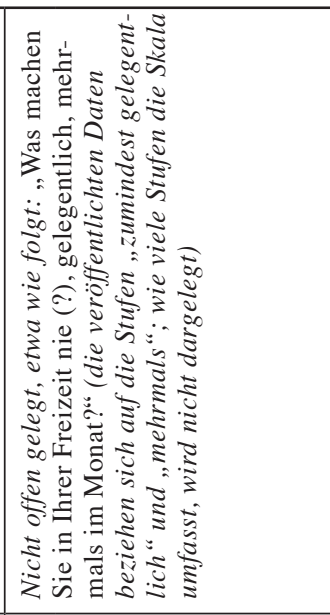 & 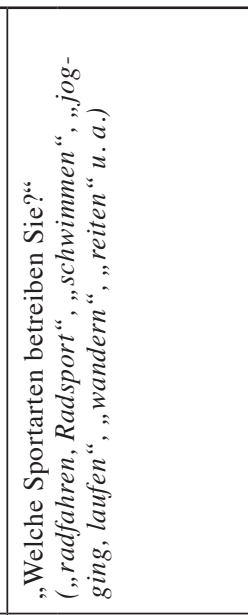 & 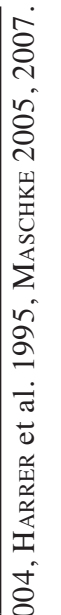 \\
\hline 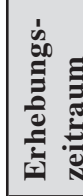 & & 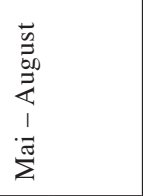 & 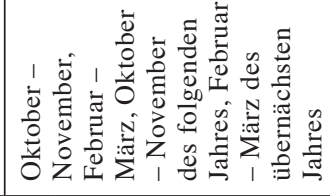 & 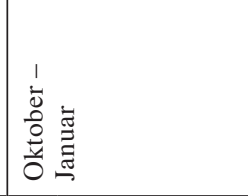 & \\
\hline 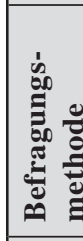 & & 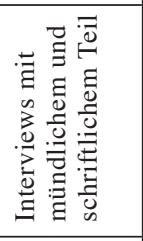 & 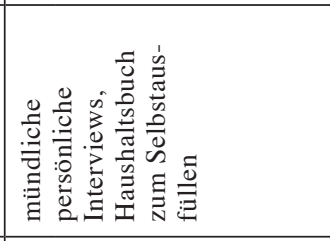 & 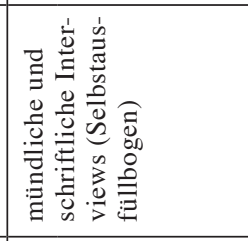 & 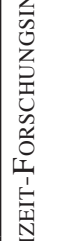 \\
\hline 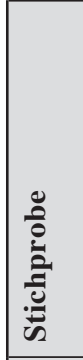 & & 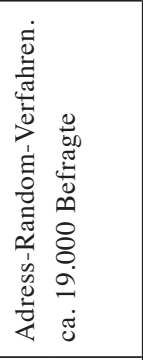 & 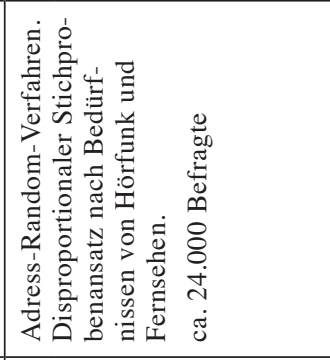 & 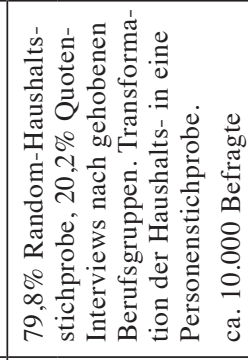 & 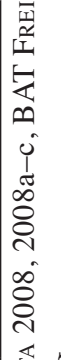 \\
\hline 它 & & 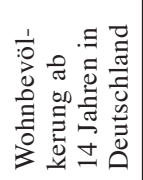 & 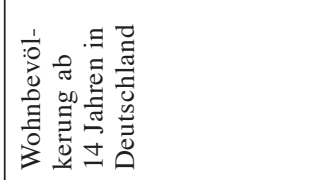 & 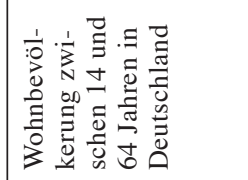 & \\
\hline 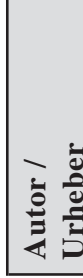 & 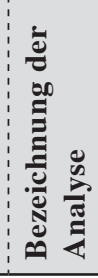 & 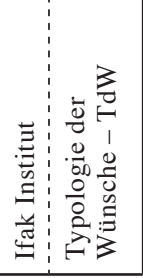 & 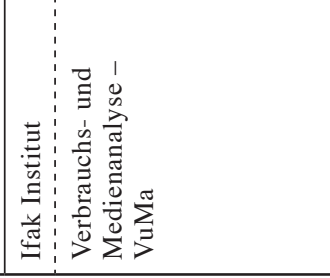 & 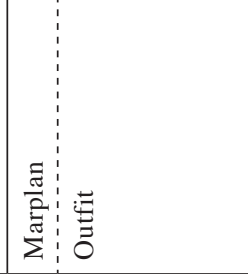 & \\
\hline
\end{tabular}


ren Erhebungen den Befragten überlassen, sie wird aber wohl über die vorgegebene Assoziation mit Sport beeinflusst.) Gleiches gilt auch für den Freizeit-Monitor bis 2003, der aufgrund der Eingrenzung der Fragestellung auf den Zeitraum der Woche vor der Befragung trotz der gemeinsamen Erhebung der Aktivitäten Spaziergänge und Wanderungen auf niedrigere Werte kommt als die AWA.

Während sich die Divergenzen im Querschnitt der Institute plausibel erklären lassen, fehlen einleuchtende Ursachen für die tendenzielle Gegenläufigkeit der Längsschnittdaten. Auf einen „Wandertrend“ deuten nur die AWA-Werte von 1999 bis 2005. Die Ergebnisse des dwif verweisen eher auf eine tendenziell gesunkene Bedeutung des Wanderns zwischen 1993 und 2006. Auch die Zeitreihe des Freizeit-Monitors bis 2003 kann keinen Boom belegen.

\subsubsection{Weitere „Outdoor“-Freizeitaktivitäten im Vergleich}

In Abbildung 2 ist die Freizeitaktivität Wandern weiteren Freizeitaktivitäten gegenübergestellt, die der AWA zufolge 2007 von mehr als 20\% der Deutschen ausgeübt werden. Das Bergsteigen als Freizeitaktivität wurde wegen der Ähnlichkeit des Bewegungsablaufs mit dem des Wanderns in die Abbildung aufgenommen, obwohl es diesem Kriterium nicht genügt. Dem Freizeit-Monitor zufolge gehen nun mehr Deutsche in ihrer Freizeit dem Schwimmen und Radfahren nach als dem Wandern. Mindestens einmal pro Woche fahren 38\% der Deutschen in ihrer Freizeit Rad. Auch wenn 78\% der Deutschen schwimmen, so machen dies vergleichsweise geringe 7\% einmal in der Woche oder öfter. Damit ist die Zahl der sehr regelmäßigen Wanderer (6\%) so gut wie gleich hoch wie die der sehr regelmäßigen Schwimmer (STIFTUnG FÜr ZukunftSFRagen 2008). Der Freizeit-Monitor erhebt weder Daten zum Jogging noch zum Bergsteigen. Zahlen hierfür liegen von der AWA und von Outfit vor; in der Outfit-Erhebung wird nach Sportaktivitäten gefragt. Joggen ist demnach die drittbeliebteste Sportaktivität und mit 28\% etwas öfter genannt als das Wandern (STATISTA 2008c). Nur 2\% der Deutschen geben Bergsteigen als ausgeübten Sport an (Statista 2008 c). Gemäß der AWA bezeichnen allerdings $12 \%$ der Deutschen das Bergsteigen als eine ihrer ausgeübten Freizeitaktivitäten (IFD 2007). Wie in Kapitel 4.1.2 im Bezug auf das Wandern bereits erwähnt und erklärt, sind die Outfit-Werte niedriger als die Zahlen des Freizeit-Monitors (2007) und der AWA. Dies gilt analog für das Schwimmen, Bergsteigen und für das Jogging, wenn auch beim Jogging in weitaus geringerem Maße - so joggen der AWA zufolge 31\% der Deutschen (IFD 2007). Dies dürfte daran liegen, dass das Jogging als „Outdoor“-Aktivität gewisse körperliche und gegebenenfalls auch mentale Ressourcen erfordert, die Eintrittsbarrieren damit relativ hoch sind, und das Joggen daher entweder gemacht wird oder nicht.

Folgt man den AWA-Werten, so fällt auf, dass Wandern nach dem Schwimmen auf Platz zwei liegt. Zu beachten ist allerdings, dass die AWA nicht das Radfahren, sondern nur das Mountainbiking als Freizeitaktivität erhebt. Festzuhalten ist resümierend, dass das Wandern zu den beliebtesten Freizeitaktivitäten der Deutschen zählt; Deutsche schwimmen aber häufiger und fahren öfter Rad. 


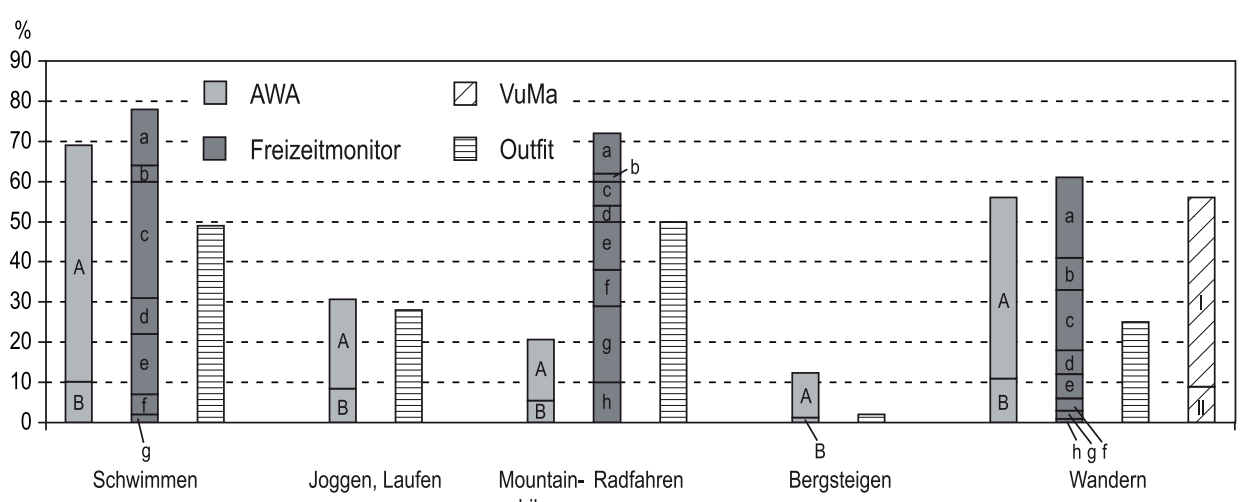

A: ab und zu, B: häufig

a: seltener als einmal jährlich, b: vielleicht einmal jährlich, c: mehrmals im Jahr, d: etwa einmal pro Monat, e: mehrmals im Monat, f: etwa einmal pro Woche, g: mehrmals in der Woche; h: täglich

I: gelegentlich, II: mehrmals im Monat

Quellen: IfD 2007, Statista 2008, 2008c, Stiftung FÜr Zukunftsfragen 2008. Eigene Zusammenstellung.

Abb. 2: Weitere „Outdoor“-Freizeitaktivitäten 2007 im Spiegel der Marktforschung

\subsection{Stellenwert der Urlaubsaktivität Wandern und Trekking}

\subsubsection{Resultate}

Wie verhält es sich bei den Urlaubsaktivitäten? In den Medien (z.B. TIP DER WOCHE 2004) wird mit Verweis auf BRÄMER von einem Anteil von 70\% der Deutschen berichtet, der im Urlaub wandert. BräMER (2005) nennt diese Zahl ohne Angabe der Quelle und des Referenzjahres. ${ }^{5)}$ Die Ergebnisse der in der deutschen Tourismusbranche anerkannten Reiseanalyse (FUR diverse Jahrgänge, STENGER 1998) weichen hiervon deutlich ab. Bei dieser touristischen Großerhebung wird gefragt, welche Aktivitäten im Urlaub des vergangenen Jahres (Fragestellung bis 1999) bzw. in den Urlauben der letzten drei Jahre ausgeübt wurden (vgl. Tab. 2). Im Reisejahr 1990 wanderten demzufolge $49 \%$ der Deutschen, $199154 \%{ }^{6}$ ) (die Häufigkeit wurde nicht erhoben; StfT 1991, 1992). Für den Zeitabschnitt 1996-1998 gaben 48\% der befragten Personen an, in den Ferien sehr häufig oder häufig gewandert zu sein, für die Zeiträume 1998-2000, 2000-2002 bzw. 2001-2003 und 2002-2004 beläuft sich der Wert nur noch auf 36\%, $31 \%, 35 \%$ bzw. 33\% (vgl. Abb. 3; FUR 2006, 2008) und nimmt seitdem weiter leicht

\footnotetext{
5) BRÄMER (E-mail-Mitteilung 2009) ermittelt diesen Wert in den deutschen Mittelgebirgen. Eine Hochrechnung ist damit allenfalls auf die Grundgesamtheit der deutschen Mittelgebirgstouristen zulässig.

6) Für die Reisejahre 1990 und 1991 werden die Ergebnisse nach Alten und Neuen Bundesländern aufgeschlüsselt. Der Stellenwert des Wanderns ist in den Neuen Bundesländern jeweils erheblich höher als in den Alten Bundesländern: 64\% bzw. 66\% der Ostdeutschen, jedoch nur 45\% bzw. 50\% der Westdeutschen gaben an, in den Ferien gewandert zu sein (StfT 1991, 1992).
} 


\begin{tabular}{|c|c|c|c|}
\hline 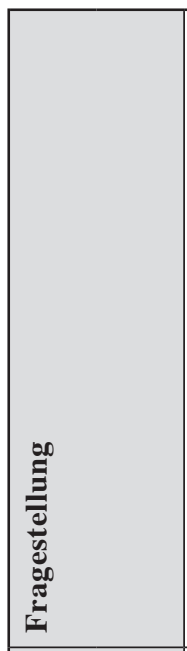 & 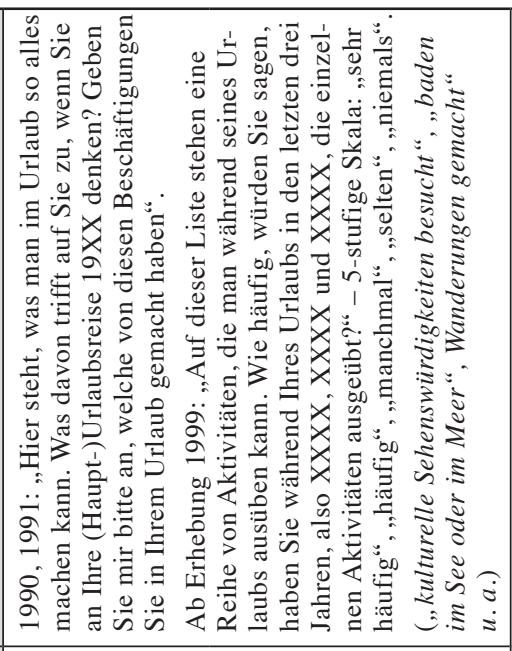 & 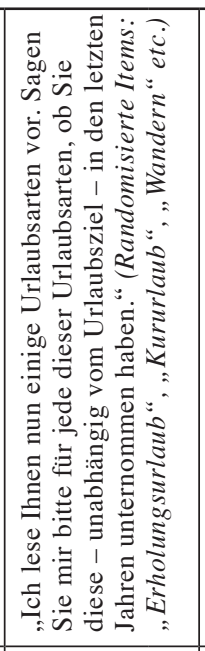 & 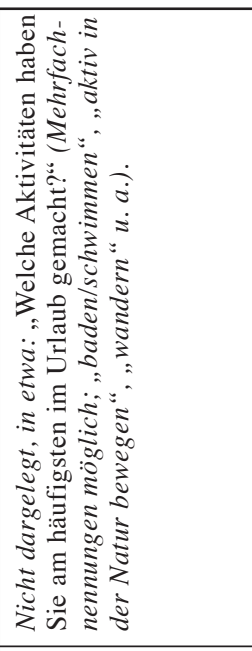 \\
\hline 尊 & \begin{tabular}{|l} 
离 \\
善
\end{tabular} & 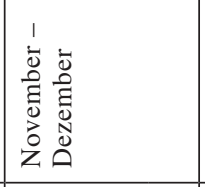 & 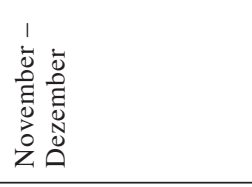 \\
\hline 总 & 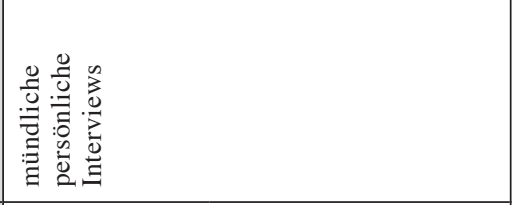 & 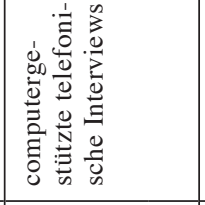 & 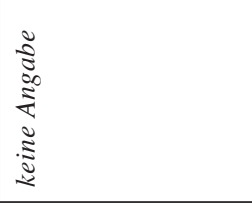 \\
\hline to & 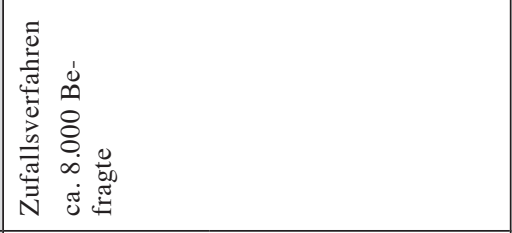 & 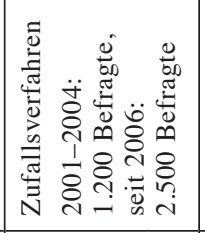 & 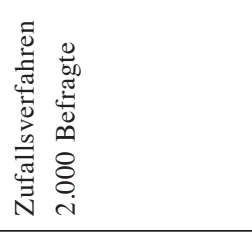 \\
\hline 吾 & 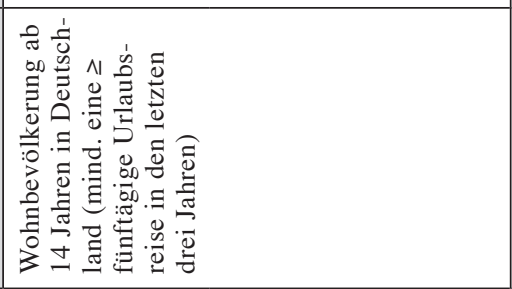 & 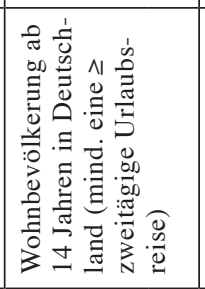 & 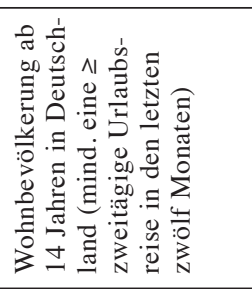 \\
\hline 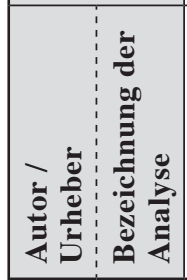 & 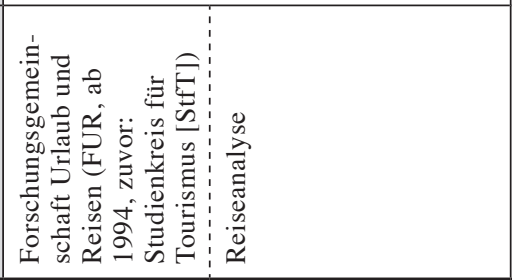 & 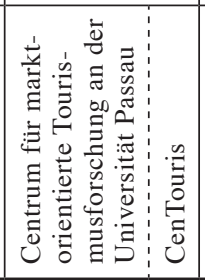 & 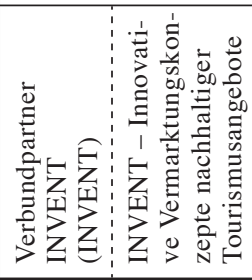 \\
\hline
\end{tabular}


„Megatrend“ Wandern und Trekking?

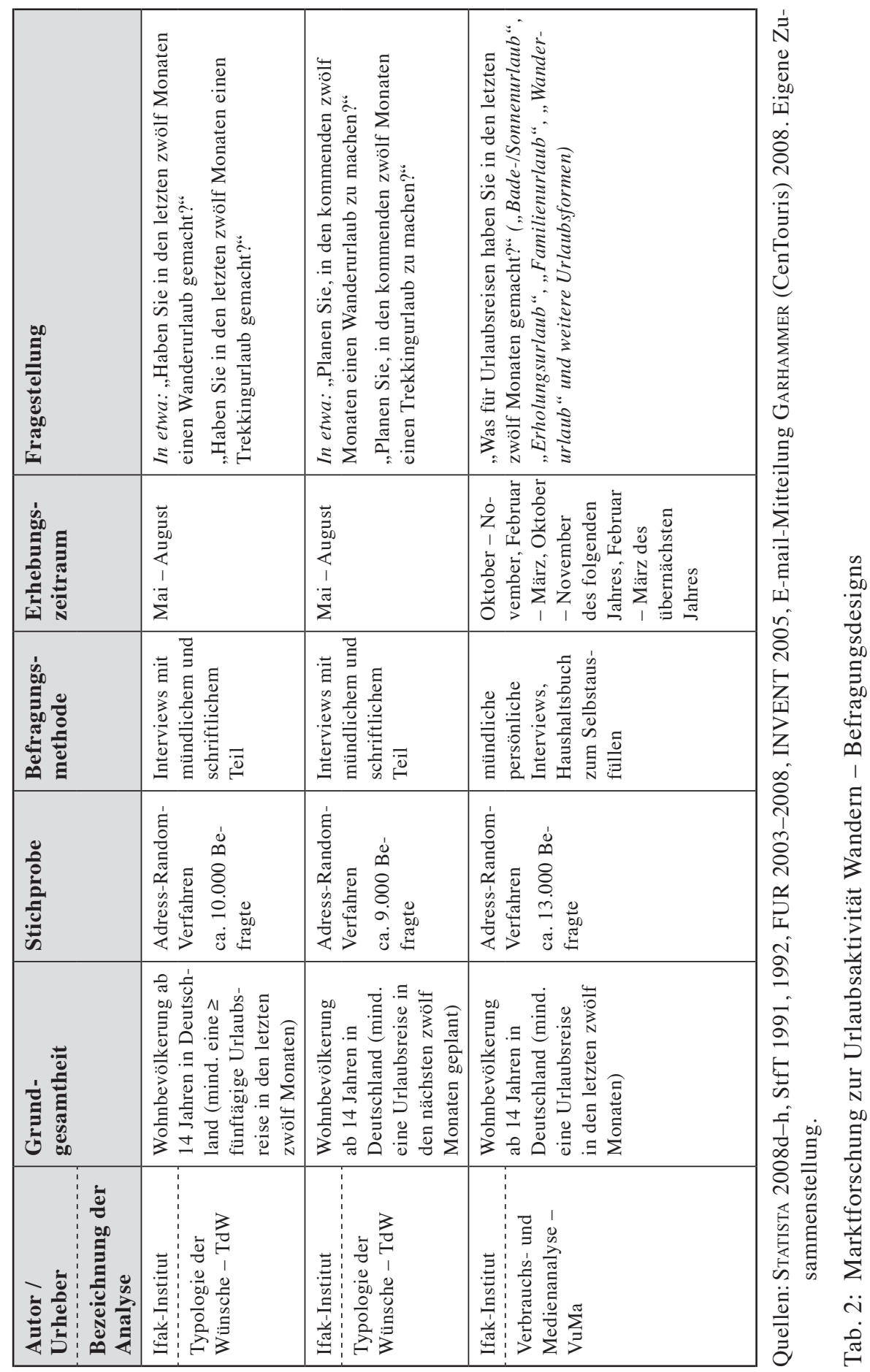




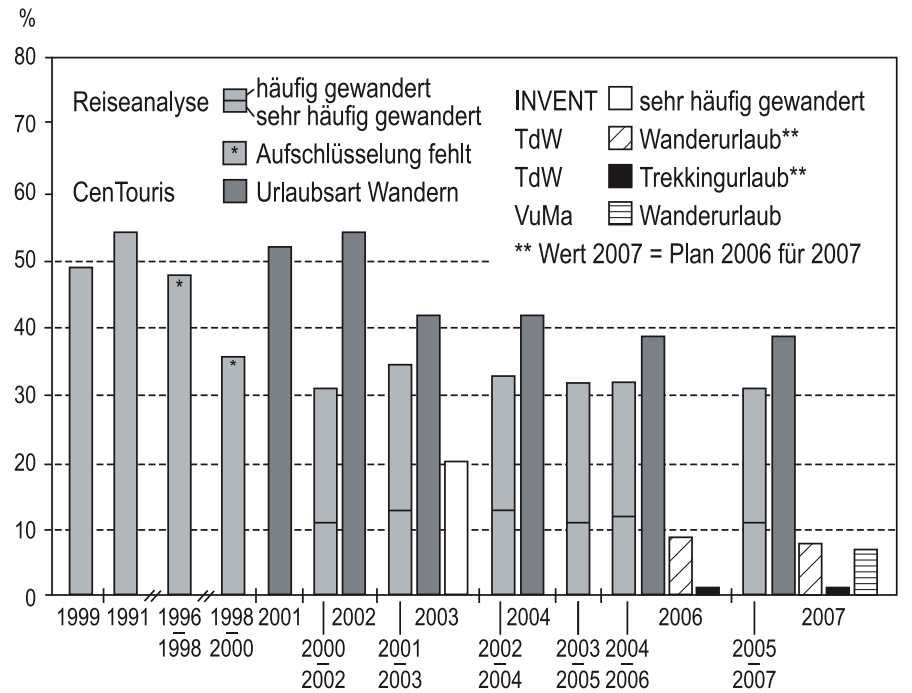

Reiseanalyse: Erhebungsbeginn unbekannt (vor 1990), Werte vor 1990, 1992-1995, 1997, 1999 in der gesis-Bibliothek verschollen; INVENT: einmalige Erhebung 2003; TdW, VuMa: Erhebungsbeginn unbekannt, keine Kenntnis über fehlende Werte; CENTourIs: Erhebungen seit 2001, keine Erhebung 2005.

Quellen: Statista 2008d-h, StfT 1991, 1992, Becker 2000, Leder 2003, FUR 2003-2008, INVENT 2005, E-mail-Mitteilung GARHAMMER (CenTouris) 2008. Eigene Zusammenstellung und eigener Entwurf.

Abb. 3: Entwicklung der Urlaubsaktivität Wandern / von Wanderurlauben im Spiegel der Marktforschung

ab: 2003-2005 wanderten 32\% im Urlaub, 2004-2006 sowie 2005-2007 nur noch 31\% (FUR 2006, FUR 2008).

Die Untersuchung von INVENT, bei der nach den häufigsten Urlaubsaktivitäten (mit der Möglichkeit von Mehrfachnennungen) gefragt wurde, kommt auf von der Größenordnung in etwa kompatible Resultate (BFN \& DTV 2005, S. 7; INVENT 2005, S. 15). 34\% der Deutschen bewegen sich im Urlaub aktiv in der Natur, $38 \%$ bummeln und gehen spazieren, 20\% geben an, sehr häufig gewandert zu sein. CENTouris (2008) stellt wie die FUR einen Rückgang der Wanderneigung fest, wenngleich auf höherem Niveau: Wandern als „Urlaubsart“ unternahmen „,in den letzten Jahren“ 2007 39\% der Deutschen, 2001 waren es noch 52\%. Die VuMa gibt 2007 hingegen an, dass nur $7 \%$ der Deutschen, die eine Urlaubsreise unternommen haben, einen Wanderurlaub wählten (STATISTA 2008d). Auch der TdW zufolge machten 2006 geringe 9\% der Urlauber der letzten zwölf Monate einen Wanderurlaub (STATISTA 2008e), und nur $8 \%$ der Befragten, die für die nächsten zwölf Monate einen Urlaub planten, hatten vor, eine Wanderreise zu unternehmen (Statista 2008f.). In der TdW-Marktanalyse wurde auch die Beliebtheit des „Trekkingurlaubs“ erhoben. Nur 1\% der Deutschen unternahm bei der Befragung 2006 in den letzten zwölf Monaten einen Trekkingurlaub; gleich wenige planten einen Trekkingurlaub für das Jahr darauf (STAтISTA 2008g, 2008h). In allen Erhebungen wurde nicht zwischen in- und ausländischen Zielgebieten differenziert. 


\subsubsection{Diskussion der Ergebnisse}

Gleich den Erhebungen zur Freizeitaktivität Wandern sind die unterschiedlichen Ergebnisse der auf den Urlaub bezogenen Erhebungen durch die Fragestellungen bedingt. INVENT erhebt die häufigsten Urlaubsaktivitäten, während die Reiseanalyse zur Antwort eine mehrstufige Skala anbietet. Bei der TdW und der VuMa wird die Durchführung (und Planung) eines „Wanderurlaubs“ erhoben. Die Interpretation des Begriffs bleibt dabei (wie bei den übrigen Erhebungen der Begriff des „Wanderns“) den Befragten überlassen; dabei dürfte der Terminus „Wanderurlaub“ so aufgefasst werden, dass die Hauptbeschäftigung im Urlaub das Wandern im engeren Wortsinn war (während bei einer Frage nach der Aktivität „Wandern“ die Befragten wohl einerseits darunter eventuell Spaziergänge subsumieren, andererseits das Wandern nicht als den Urlaub ausschließlich definierende Größe interpretieren). Bei CenTouris wird mit einer „Ja-Nein“-Fragestellung erhoben, ob „Wandern“ als „Urlaubsart“ „,in den letzten Jahren unternommen“ wurde, wobei der Zeitraum der Urlaubsreisen nicht eingeschränkt ist. Die Werte von CenTouris dürften einerseits deshalb höher liegen als die der TdW und der VuMa. Zudem wird nicht explizit von einem „Wanderurlaub“ gesprochen, sondern von der „Urlaubsart“ Wandern. Die höheren Werte von CenTouris im Vergleich zur Reiseanalyse liegen wohl darin begründet, dass nicht die Häufigkeit abgefragt wurde.

Festzuhalten ist jedenfalls, dass weder Wander- noch Trekkingurlaube im engeren Sinn in der Bevölkerung sehr populär sind, auch wenn die „Renaissance des Wanderns" regelmäßig mit Weitwanderungen und Trekking in Verbindung gebracht wird (GERBERT 2005, StePhAN 2007). Eine Renaissance kann auch im Längsschnittvergleich nicht ausgemacht werden. Aus der Reiseanalyse resultieren ein Rückgang bzw. eine Stagnation des Stellenwerts des Wanderns; auch im Zeitraum 1999-2005, für den die AWA eine Zunahme des Wanderns als Freizeitaktivität feststellt, gehen die Werte zurück. Ebenso deuten die Zeitreihenwerte von CenTouris auf einen Rückgang des „Wanderns als Urlaubsart“. Über mögliche Gründe für die rückläufige bzw. stagnierende Entwicklung des Wanderns als Urlaubsaktivität können nur Vermutungen angestellt werden. Der Reiseanalyse zufolge folgt auch das Radfahren im Urlaub einem leichten Abwärtstrend. Dennoch werden Urlauber nicht passiver - der Anteil derer, die in den Ferien ,viel schlafen, ausruhen“, ist über die Jahre hinweg konstant (StfT 1991, FUR 2003-2008).

Insgesamt mag auffallen, dass die Zahlen für das Wandern im Urlaub niedriger sind als die für das Wandern in der Freizeit (vgl. Abb. 1 und 3). Aus den vorliegenden Daten kann jedoch nicht vorbehaltlos auf einen geringeren Stellenwert des Ferienwanderns geschlossen werden, da sich die Daten nur schwer vergleichen lassen. Den Fragestellungen der Freizeitaktivitäten-Erhebungen ähnelt die der Reiseanalyse ab 1999 am meisten (vgl. Tab. 1 und 2). Ihre Skala für die Antworten ist fünfstufig, wobei allerdings in den veröffentlichten Daten nur die Stufen „,sehr häufig“ und ,häufig“ für die Beliebtheit einer Aktivität zusammengefasst werden. (Daten zu den Antwortmöglichkeiten „manchmal“ und „selten“ werden nicht veröffentlicht.) Dieser Unterschied könnte die Wertedifferenzen zwischen der Reiseanalyse und z.B. der AWA erklären. 


\subsubsection{Weitere „Outdoor"-Urlaubsaktivitäten im Vergleich}

Zur Einordnung des Stellenwerts des Wanderns im Urlaub sollen die aktuellsten Werte (2007, bei der TdW die Pläne für 2007) mit denen für die Urlaubsbeschäftigungen Schwimmen bzw. Baden und Radfahren verglichen werden. (Weder Jogging noch Bergsteigen werden in den Urlaubsaktivitäten-Analysen erhoben.) Abbildung 4 zeigt, dass die Deutschen im Urlaub deutlich lieber baden als wandern. Das Radfahren spielt im Urlaub hingegen im Vergleich zur Alltagsfreizeit eine weitgehend vernachlässigbare Rolle. Der Reiseanalyse zufolge zählen Wanderungen im Urlaub zu den am vierthäufigsten ausgeübten landschaftsbezogenen Aktivitäten - nach „Ausflügen in die Umgebung“, dem in Abbildung 4 aufgeführten „Baden im See oder Meer“ und dem Besuch von „Naturattraktionen“(FUR 2008).

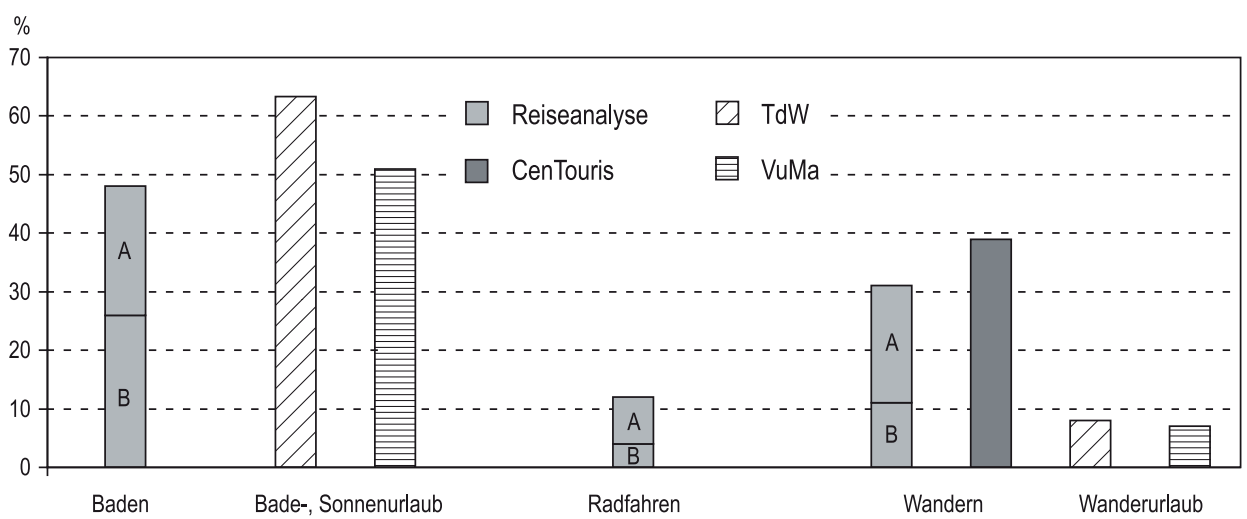

A: häufig, B: sehr häufig

Quellen: FUR 2008, Statista 2008d, 2008f, 2009, E-mail-Mitteilung Garhammer (CenTouris) 2008. Eigene Zusammenstellung.

Abb. 4: Weitere „Outdoor“-Urlaubsaktivitäten 2007 im Spiegel der Marktforschung

\section{Wandern und Trekking im Spiegel möglicher Indikatoren}

\subsection{Medienpräsenz des Wanderns und Trekkings}

Ungeachtet der wenig konsistenten Datenlage lässt sich in den letzten Jahren eine hohe Medienpräsenz des „Trendsports Wandern“ beobachten (vgl. SEIFERT-RösING 2006 , S. 5; Prantl 2007). Allein in den Print- und Onlinemedien nehmen sich unterschiedliche Mediengenres (internationale, überregionale und regionale Zeitungen, Polit-, Verbraucherschutz- und Wirtschaftsmagazine, Frauenzeitschriften, Kundenzeitungen etc.) des Wanderns und insbesondere des Wandertrends an. Dieser wird häufig mit Werten der AWA belegt; teilweise fehlen aber auch Belege für die Aussage (SZ 2003, 
Tip der Woche 2004, Brunner 2004, Gerbert 2005, NZZ 2005, Werner 2005, LIFEline 2007, Prantl 2007, Stephan 2007, HaAs 2008, Petermann 2008). Die Verfasserin stieß nur auf einen einzigen Beitrag, in dem auch andere Marktforschungsdaten als die der AWA zitiert werden. So nimmt PrANTL (2007) neben der AWA auch auf die Reiseanalyse Bezug und stellt dabei die Widersprüchlichkeit der Ergebnisse fest, konstatiert aber gleichzeitig die Popularität der Annahme eines Wandertrends in der öffentlichen Wahrnehmung. Eine Analyse des Online-Archivs der Süddeutschen Zeitung (http:// archiv.sueddeutsche.de/sueddz/) der Jahre 1992 bis Ende Juli 2009 zeigt zwar, dass in den 1990er Jahren jährlich mehr Beiträge, die sich mit Wanderangeboten befassten, ${ }^{7)}$ erschienen sind als in den Jahren seit der Jahrtausendwende. Gleichzeitig sind aber seit 200123 Beiträge publiziert worden, die das Wandern in Zusammenhang mit Trends bringen, von 1992 bis 2000 jedoch nur acht. Ähnliche Ergebnisse zeigt eine Prüfung des Online-Archivs der Frankfurter Allgemeinen Zeitung (http://fazarchiv. faz.net/) der Jahre 1994 bis Ende Juli 2009. ${ }^{8}$ B Bis Ende 2001 befassten sich 58 Beiträge mit Wandern, von 2002 bis Sommer 2009 56. Meldungen, die eine „neue Lust“ am Wandern ausmachen, finden sich nur im ersten Jahrzehnt des 21. Jhs.

Damit hat sich primär die Form der Berichterstattung geändert; ein Anstieg der Medienpräsenz des „Wanderns“ per se lässt sich nicht erkennen.

„Wo die Meldung vom Trendsport Wandern ihren Ursprung hatte, lässt sich heute kaum noch nachvollziehen. Sie war auf einmal da, ungefähr so wie der Klimawandel, Dieter Bohlen oder das Nordic Walking. Irgendwann entwickelte sie eine Eigendynamik, verfestigte sich zur unumstößlichen Tatsache, wurde gierig aufgegriffen von den PR-Maschinisten und der Sportartikelindustrie und pflanzte sich von dort aus über Kampagnen bis zum Kunden fort. Auf einmal war Wandern überall einfach richtig angesagt“" (PRANTL 2007, S. 38).

In Kapitel 5.5 wird möglichen Ursachen für die geänderte Berichterstattung im Kontext der Positionierung touristischer Destinationen nachgegangen.

\subsection{Themenschwerpunkte von Messen und Tagungen}

Neumeyer \& Dicks (2004) sehen in der Gründung einer spezifischen Wander- und Trekkingmesse, der „TourNatur“ in Düsseldorf, ein weiteres Anzeichen für eine Renaissance des Wanderns. Seit 2003 findet diese Messe statt, die seit 2004 im Mittel 30.000 Gäste anzieht (MESSE DüssEldorf 2006, 2007, 2008). Blendet man auch im Folgenden zunächst aus, was das Indikatum eines Indikators „Messen“ darstellt, und analysiert man die Themenschwerpunkte und Angebote der nach der Ausstellerzahl größten Tourismusmessen in Deutschland, so lässt sich Folgendes feststellen: Seit

\footnotetext{
7) Recherchiert wurde mittels des Suchbegriffs „,wandern“ in den Titeln der SZ-Beiträge. Alle Treffer wurden anhand des vollständigen Titels und dem Artikel-Anfang daraufhin überprüft, ob sich das „Wandern“ auf die Bewegung per pedes in der Freizeit bezog. Da wegen der großen Menge an Treffern bei der Suche nach „wandern“ in den Titeln und Artikeln (6.819 Treffer) keine vollständige Analyse der Artikel durchgeführt wurde, könnte es möglich sein, dass sich die Abnahme des „Wanderns“ nur in den Titeln vollzog.

8) Die Suche erfolgte analog zur Recherche im SZ-Archiv (vgl. Fußnote 7).
} 
2004 setzen auch die Internationale Tourismusbörse (ITB) in Berlin und die „C-B-R Freizeit und Reisen" (seit 2009 unter dem Namen f.re.e) in München auf das Segment „Wandern“ und etablieren entsprechende thematische Schwerpunkte mit Podiumsdiskussionen und Fachtagungen (vgl. ITB 2006, 2008, C-B-R Freizeit und ReISEN 2005, Messe München GmbH 2009). Auch die Urlaubsmesse CMT in Stuttgart bietet 2008 und 2009 Sonderausstellungen zum Wandertourismus an (Messe Stuttgart 2008, 2009). Im Jahr 2006 präsentierten nahezu alle Reisemessen in Deutschland „Wanderund Outdoorangebote“" (WandERMAGAZIN 2006, S. 37).

Der „Trendsport Wandern“ avancierte inzwischen auch zum Thema von Tagungen nicht nur von Wander- und Bergsportverbänden (verschiedene Konferenzen des Deutschen Alpenvereins und des Deutschen Wanderverbands), sondern auch von Institutionen der Erwachsenenbildung (z.B. Thomas-Morus-Akademie 2005) und Berufsverbänden (DVAG 2009).

\subsection{Das „Outdoor“-Segment des Sportartikelmarkts}

Vor allem auch an den „Outdoorangeboten“ bei Bekleidung und Ausrüstung wird der Wandertrend in den Medien und in der deutschen wissenschaftlichen Literatur festgemacht (vgl. z.B. BRÄMer 2003, S. 3; Neumeyer \& Dicks 2004, S. 285). Der Handel mit Funktionsbekleidung und -ausrüstung gilt als das am stärksten wachsende Segment im Sportartikelmarkt (Verband Deutscher SportfachHandel 2008). „Trotz Konjunkturflaute wuchs der Markt für ,Outdoor'-Ausrüstung (ohne Fahrräder) von 2001 bis 2004 um mehr als sieben Prozent. Mittlerweile kann man sich sogar in TchiboKaffeeläden und bei Lebensmitteldiscountern einkleiden" (GERBERT 2005, S. 118). In Deutschland sei jeder Zweite an „Outdoor“-Kleidung interessiert (Focus 2005, S. 19).

Die Annahme, der „Outdoor“-Ausstattungsmarkt könne als Indikator für die Beliebtheit des Wanderns dienen, trägt allerdings nicht der Tatsache Rechnung, dass sogenannte Funktionskleidung inzwischen als alltagstauglich gilt und damit nicht allein zum Wandern oder Trekking verwendet wird (Focus 2005, S. 18; PetermanN 2008, Verband Deutscher Sportfachhandel 2008a). In den vergangenen Jahren wurde der Sport aufgrund neuer Körperbilder und -ideale „prägendes Lebensstil-Merkmal“ (Focus 2005, S. 18). So wird zum Teil eine sportliche Ausrüstung und Kleidung zur Identifikation - auch im Alltag - verwendet, unabhängig davon, ob alle spezifischen Funktionen von „Outdoor“-Bekleidung dort wirklich benötigt werden.

Angebots- und Nachfrageseite beeinflussen sich bei der Trendgenerierung wechselseitig, wobei vielfach den Herstellern zugeschrieben wird, Trends künstlich zu verstärken. Dass , „s]pezielle Outfits und Marken im ,Outdoor'-Bereich [...] für Freiluft Fans und Globetrotter einfach ,Kult"“ wurden (Focus 2005, S. 18), liegt auch daran, dass die Anbieter die Nachfrager mit modischem und alltagstauglichem Design abholen konnten (vgl. E-mail-Mitteilung JACK WoLFSKIN 2008). 


\subsection{Wander- und Trekkingwege - die touristische Angebotsseite in Deutschland und Österreich}

Die Popularität des Wanderns in der medialen Wahrnehmung schlägt sich in der Positionierung touristischer Destinationen in Deutschland und Österreich über Fernwander- und Trekkingwege nieder. In Deutschland wird bei der Vermarktung zum Teil auf bestehende Wege zurückgegriffen, zum Teil werden in den letzten Jahren neue mit narrativen Themenbezügen geschaffen, wobei vorhandene Wege oft ausgebaut und verknüpft werden. Dazu zählen Weitwanderwege wie z.B. Rothaarsteig, Rheinsteig, Frankenweg, Harzer Hexen-Steig, insbesondere auch Pilgerwege wie eine unüberschaubare Zahl an neuen Jakobswegzubringern (vgl. HeIDEMAnN 2004, GERBERT 2007).

Die Eröffnung neuer Weitwanderwege wurde angetrieben durch das Projekt „Wanderbares Deutschland“ des Deutschen Tourismusverbands und des Deutschen Wanderverbands mit finanzieller Unterstützung durch das deutsche Bundesministerium für Wirtschaft und Arbeit von 2001 bis 2003 (Deutscher W Anderverband 2004). Neben einem Webauftritt mit Informationen zu über 100 Fernwanderwegen in Deutschland (Deutscher Wanderverband 2006) brachte das Projekt 2004 auch ein Gütesiegel für Wanderwege hervor. Gegenwärtig sind 41 Wege mit diesem Prädikat ausgezeichnet worden (Deutscher W Anderverband 2008). Die Zertifizierungskriterien beziehen sich auf das Wegeformat, das Wanderleitsystem, Natur und Kultur (Deutscher W ANDERVERBAND 2004). Wohl wegen der inzwischen vorliegenden Ubiquität und der fehlenden Alleinstellung durch Weitwanderwege bemühen sich Touristiker um eine weitere Differenzierung, sodass es inzwischen mindestens fünf verschiedene Qualität versprechende Labels gibt (Gerbert 2007, Messe Düsseldorf 2008).

Frequenzzahlen der Weitwanderwege liegen nicht vor; sehr hohe Frequenzen sind aber angesichts des äußerst geringen Anteils an Trekkingurlaubern in der Bevölkerung Deutschlands nicht zu erwarten. Während in einigen Regionen mit den Weitwanderwegen das Marktsegment der Weitwanderer angepeilt wird, dienen die Wege in anderen Regionen primär zur Image- und Markenbildung.

Dies wird in Österreich sehr deutlich: So setzt sich Tirol mit einem „Adlerweg“ „auf den Schwingen des Adlers“ in Szene (SchNöLl 2005) und bemüht sich damit um eine Positionierung der gesamten Destination als Bergregion, die eine Alteritätserfahrung im Urlaub durch die majestätische Perspektivierung „,von oben“ ermöglicht. Damit messen auch in Österreich Tourismusorganisationen dem Wandern, Bergwandern und Trekking für den Sommertourismus große Marktpotenziale bei; entsprechende Bemühungen werden zum Beispiel an der digitalen Erfassung und Webpräsentation des Vorarlberger Wanderwegenetzes (LAND VoRARLBERG 2004) oder an der Positionierung der Zielgebiete seitens der Tourismusorganisationen sichtbar. So sind die Tourismusprospekte mit Slogans überschrieben wie „Du wirst Dich wandern“ (TIROL INFO 2005), „Raus aufs Land: Niederösterreich“ (NIEDERÖSTERREICH INFORMATION 2005) oder „Niederösterreich neu erwandern“ (NIEDERÖSTERREICH Werbung GMBH 2009), wobei das Titelbild 2005 muskulöse Beine (in traditionellen Bergstiefeln und Kniebundhosen) auf einem Gipfel vor einem Gebirgspanorama präsentierte.

Die landesweite Tourismusorganisation „Österreich-Werbung“ bemüht sich in den letzten Jahren stark um das Marktsegment der Wanderer und lancierte so auf ihrer 
Webpräsenz einen Wandertypentest, um dem potenziellen Gast auf ihn zugeschnittene Angebote offerieren zu können (ÖsTERREICH WERBUNG 2009). ${ }^{9}$

\subsection{Diskussion der Indikatoren und Thesen zur Entwicklung der Variablen}

Sind die in Kapitel 5.1 bis 5.4 beschriebenen Sachverhalte Indikatoren für die Entwicklung der wander- und trekkingtouristischen Nachfrage? Der Indikator „Medienpräsenz" zeigt die Konnotation des Wanderns mit einem Trend an, der Indikator Messen und Tagungen deutet gleich dem Indikator der Positionierung touristischer Destinationen mittels Wander- und Trekkingwegen auf ein gestiegenes Interesse der Angebotsseite am Marktsegment Wandern und Trekking. Wie in Kapitel 4 dargelegt, können Erhebungen der Marktforschungsinstitute - sieht man von der AWA für den Zeitraum von 1999 bis $2005 \mathrm{ab}$ - weder für das Wandern als Freizeitbeschäftigung noch für das Wandern und Trekking als Urlaubsaktivität ein Wachstum der Nachfrage (wohl aber - abgesehen vom Trekking - einen relativ hohen Stellenwert dieser Aktivitäten) feststellen. Damit verläuft der Anstieg der Bedeutung, die seitens der touristischen Angebotsseite dem Wandern und Trekking beigemessen wird, tendenziell unabhängig von der Entwicklung der Nachfrage. Weshalb aber hat die touristische Angebotsseite den Wander- und Trekkingtourismus neu entdeckt?

Die Ursachen dürften an der kritischen Lage deutscher und österreichischer Destinationen liegen, die sich in ihrem Lebenszyklus (vgl. BUtLer 1980, S. 7) in einer Stagnations-, teilweise auch Niedergangsphase befinden - BäTZING (2003, S. 159) zufolge seit Mitte der 1980er Jahre, Јов (2005, S. 128) zufolge seit Mitte der 1990er Jahre. In der wissenschaftlichen Literatur werden folgende Punkte als Gründe für die Krise genannt:

- Wandel des Tourismusmarkts von einem Verkäufer- zu einem Käufermarkt;

- wachsende Ansprüche der Käufer aufgrund zunehmender Reiseerfahrung;

- Assoziation der Alpen (primär im Sommer) als biederes und tendenziell langweiliges Urlaubsziel (vgl. Bachleitner \& Weichbold 2002);

- gesunkene Flugpreise und Liberalisierung des Kapital-, Güter- und Personenverkehrs;

- damit globale Konkurrenz;

- zunächst Überforderung der lokalen und regionalen Tourismusorganisationen mit der geänderten Wettbewerbssituation;

- fehlende Handlungsressourcen der touristischen Leistungsträger, meist Mikro- und Kleinunternehmen, für eine erfolgreiche Neupositionierung (vgl. Vogt 2008).

\footnotetext{
9) Auch in der Schweiz steigen in den letzten Jahren die Zahl der Trekkingwege und vor allem die Vermarktung dieser Wege an. Kundert (2006, S. 35f.) bemerkt die Neuheit ,authentische“ Naturerlebnisse versprechende Trekkingwege zu vermarkten: „Fernwanderwege aller Art fristeten in der Schweiz bis vor vier, fünf Jahren ein stilles Dasein, hart an der Mauerblümchengrenze. Wohl wusste man um die Existenz eines Walserweges, wohl hört man Kollegen, die auf gewissen Etappen der GTA in Italien oder auf den GRs in Frankreich unterwegs gewesen waren. Aber im Volksbewusstsein waren der Pacific Crest Trail in den USA, der Abel Tasman Coastal Track in Neuseeland [...] stärker verankert als die Tatsache, dass auch die Schweiz von grossen Fernwanderwegen durchzogen wird“.
} 
Auf der „Suche nach dem Klon“ des (trotzdem in einigen Alpenregionen aufgrund einer zielgruppenorientierten Erlebnisinszenierung erfolgreichen) Wintertourismus für den Sommertourismus (so ReISNer 2004 für Tirol) stoßen Tourismusorganisationen nun auf den Wander- und Trekkingtourismus, da das Wandern zwar nicht eine steigende, dennoch aber eine gewisse Bedeutung als Urlaubsaktivität hat. Angesichts des eher negativen Images des Wanderns (vgl. Doebeli 2003, S. 14ff., n-tv 2006 ${ }^{10}$ ) werden dem Wandern und Trekking - zum Teil wohl forciert - neue Konnotationen zugesprochen. (Berg-)Wandern wird als Antwort auf die „Überforderung des Menschen im Alltag“ angepriesen, für die „Suche nach dem individuellen Lebenssinn“ für geeignet erklärt und für stimulierend beurteilt, um sich ,in einer unvergleichlichen Bergwelt neu zu begegnen" (MARGReITER 2005). Es wird aber nicht nur mit dem Nutzen für das mentale Wohlbefinden argumentiert. SEIFERT-Rösing (2007) zieht auch die Ergebnisse einer medizinischen Studie zu den Effekten körperlicher Betätigung auf die physische Gesundheit heran (Manson et al. 1999). Die Aufwertung des Wander- und Trekkingtourismussegments seitens der touristischen Angebotsseite findet sich in den Themenschwerpunkten von Messen wieder.

Attraktiv erscheint die Ansprache der Zielgruppe der Wanderer auch wegen des wachsenden Anteils der über 50-Jährigen in der Bevölkerung. In Deutschland verschiebt sich der Altersdurchschnitt der Bevölkerung seit Jahren stetig nach oben, wobei die ,neuen Alten“ aktiver, sportlicher und kaufkräftiger sind als vorhergehende Generationen (Wenzel \& RauCH 2008). Da Wandern bei den Freizeitaktivitäten dieser Gruppe einen relativ gesicherten Stellenwert hat (bei den 50- bis 70-Jährigen sind der TdW zufolge die Anteile der Wanderer am höchsten, STATISTA 2008b) und vermutet werden kann, dass sich ältere Menschen mit dieser Freizeitbeschäftigung zumindest identifizieren können (vor allem dann, wenn dieser Aktivität nicht mehr das Image der Biederkeit anhaftet), erscheint die Strategie Gewinn versprechend, sich über Wandern und Trekking zu positionieren. Gleiches dürfte auch für Funktionskleidungshersteller gelten, die neben dem hohen Stellenwert der Sportlichkeit in der Gesellschaft auch von der Kaufkraft der „Neuen Alten“ profitieren.

Der Wandel in der Berichterstattung der Medien über das Wandern und Trekking dürfte eng mit der Wiederentdeckung des Wandersegments und der Entdeckung des Trekkingsegments seitens der touristischen Angebotsseite zusammenhängen. In einem Prozess gegenseitiger Rückkoppelungen schaukelte sich wohl das Wandern zum Trend auf, der schließlich auch von Institutionen der Erwachsenenbildung und Berufsverbänden aufgenommen wurde.

\footnotetext{
${ }^{10)}$ Einer Umfrage der Gesellschaft für Konsumforschung (zit. in n-tv 2006) zufolge bevorzugen zwar Alleinstehende als Wunschpartner sportliche Singles, aber nur $20 \%$ fanden Wanderer attraktiv (Mehrfachnennungen waren möglich).
} 


\section{Schlussfolgerungen}

Die Analyse beschäftigte sich mit der Bedeutung und Entwicklung des Wanderns und Trekkings und zeichnete diese anhand von quantitativen Nachfrageanalysen und qualitativen (vermeintlichen) Indikatoren nach. Kapitel 4.1 zeigt, dass Wandern nach Schwimmen und Radfahren die am häufigsten ausgeübte Freizeitaktivität der Deutschen ist. Etwa 8 bis $13 \%$ der Deutschen wandern regelmäßig in ihrer Freizeit, rund 41 bis $56 \%$ zumindest gelegentlich.

Die Zeitreihenwerte der Marktforschungsinstitute bestätigen allerdings nicht den häufig postulierten Trend oder gar Megatrend des Wanderns. So lässt sich zumindest keine eindeutige Entwicklung aus den Zahlen ablesen. Als Urlaubsaktivität scheint das Wandern allerdings an Bedeutung verloren zu haben. Wie in Kapitel 4.2 dargelegt, wandern der Reiseanalyse zufolge in den Jahren 2005-2007 nur 31\% der Deutschen häufig oder sehr häufig in ihren Ferien (FUR 2008), ca. zehn Jahre zuvor machten dies noch 48\% (FUR zit. in LEDER 2003). Dennoch zählen Wanderungen im Urlaub zu den vielfach ausgeübten naturbezogenen „Outdoor“-Aktivitäten.

Im Gegensatz zur quantitativ belegbaren Entwicklung des Wanderns als Freizeitund Urlaubsaktivität wird in den Medien der letzten Jahre von Wandern (teils auch Trekking) als einem Trend berichtet. Die medialen Äußerungen scheinen damit weniger ein Indikator für die quantitative Entwicklung des Wanderns als ein Indikator für die Perzeption des Wander- und Trekkingtourismussegments seitens der touristischen Angebotsseite zu sein, denn diese hat sich in den vergangenen Jahren deutlich geändert. So setzen viele ländliche Tourismusregionen vermutlich im Zuge der notwendig gewordenen Neupositionierung anlässlich der kritischen Wettbewerbslage inzwischen wieder auf das Wandern, bemühen sich um neue Konnotationen und positionieren sich mit Trekking- und Weitwanderwegen.

Was bedeuten die Ergebnisse des Beitrags für die touristische Angebotsseite? Es ist falsch, allein aufgrund eines vermeintlich wachsenden Nachfragepotenzials sehr große Hoffnungen in die Entwicklung wandertouristischer Angebote zu setzen. Vor allem bei der Lancierung von Weitwander- und Trekkingwegen ist angesichts des sehr geringen Marktanteils an Trekkingtouristen sorgfältiges Abwägen geboten. Eine nur auf Medienberichte abgestützte Abschätzung der Nachfrage kann im Zusammenspiel mit weiteren Faktoren jedenfalls zu erheblichen Fehlinvestitionen führen. So ergaben erste Evaluationen der in der Einleitung erwähnten Via Alpina im Jahr 2005 sehr geringe ökonomische Effekte des Via-Alpina-Tourismus (BERTHELOT 2006, BORSDORF 2006, Clivaz \& ToRnay 2006, Vogt et al. 2006).

Bemüht man sich mit Weitwander- und Trekkingwegen nicht um das Segment der Trekkingtouristen, sondern um das der Wanderer, so wäre eine Analyse der Wanderer nach ihren Assoziationen mit Weitwander- und Trekkingwegen für eine Überprüfung der Effektivität der Positionierung über solche Wege bei der Zielgruppe der Wanderer sinnvoll. Grundsätzlich gilt, dass angesichts der angespannten Wettbewerbslage und der eher konsolidierten Nachfrageentwicklung im Bereich des Wanderns Angebote an den Bedürfnissen und Interessen der Zielgruppe ausgerichtet sein müssen. Im vorliegenden Beitrag wurden Aspekte wie die sozioökonomischen Merkmale, das Nachfrageverhalten und die Interessen von Wanderern und Trekkingtouristen ausgeblendet. 
Für wettbewerbsfähige Angebote bedarf es aber über das Wissen zum Umfang der Nachfrage hinaus auch dieses Wissens (vgl. dazu VogT 2009).

Für die (geographische) Tourismusforschung ergibt sich als Schlussfolgerung die allgemeine Warnung davor, bei Sekundäranalysen touristischen Marktforschungsdaten blind zu vertrauen. Die Güte und Verlässlichkeit von Stichproben- und Hochrechnungsverfahren und die Objektivität und Reliabilität von Erhebungen lassen sich kaum überprüfen, da entsprechende Informationen nicht preisgegeben werden (STENGER 1998). Eine Kontrolle der Validität lässt sich aber - zumindest ansatzweise - über die konkurrente Validität mittels eines Vergleichs von Daten verschiedener Marktforschungsinstitute bewerkstelligen. Diese Forderung nach einem Datenvergleich impliziert allerdings einen hohen Aufwand, da - zumindest in Deutschland - häufig aufwändige Recherchen notwendig sind, um Daten zu erhalten. Dabei werden vor allem detailliert aufgeschlüsselte Daten nur Käufern und Auftraggebern zur Verfügung gestellt. Um irrige Thesen zu vermeiden, ist dieser Rechercheaufwand aber unumgänglich. Gewarnt sei schließlich auch vor der unbedachten Verwendung von Indikatoren wie z.B. dem der Entwicklung des „Outdoor“-Markts als Messgröße für die Beliebtheit des Wanderns und Trekkings als Freizeit- und Urlaubsaktivität. Es gilt stets zu prüfen, ob die gewählte Messgröße auch wirklich das anzeigt, was gemessen werden soll.

\section{Literaturverzeichnis}

Agricola S. (2003), Vorbemerkungen. In: THEMATA (Hrsg.), Freizeit in Deutschland 2003, S. 9-11. München - Wien, Profil-Verlag.

Ainsworth B.E. (2002), The Compendium of Physical Activities Tracking Guide. Prevention Research Center, Norman J. Arnold School of Public Health, Univ. of South Carolina. - http://prevention.sph.sc.edu/tools/docs/documents_compendium.pdf (Abrufdatum: 17.6.2010).

aLpMedia News (2006), Frankreich: Vermehrt Sommerurlaub in den Bergen? In: alpMedia News vom 27.7.2006, S. 3.

Bachleitner R., Weichbold M. (2002), Immer wieder Alpen? Anfragen zur Nachfrage im Alpentourismus. In: Luger K., Rest F. (Hrsg.), Der Alpentourismus. Entwicklungspotenziale im Spannungsfeld von Kultur, Ökonomie und Ökologie, S. 213-225. Innsbruck et al., Studien-Verlag.

BAT Freizeit-Forschungsinstitut (2002), Daten zur Freizeitforschung. Freizeit-Monitor 2002. Repräsentativbefragungen in Deutschland. Hamburg.

BAT Freizeit-Forschungsinstitut (2003), Daten zur Freizeitforschung. Freizeit-Monitor 2003. Repräsentativbefragungen in Deutschland. Hamburg.

BAT Freizeit-Forschungsinstitut (2004), Daten zur Freizeitforschung. Freizeit-Monitor 2004. Repräsentativbefragungen in Deutschland. Hamburg.

B ̈̈TZING W. (2003), Die Alpen. Geschichte und Zukunft einer europäischen Kulturlandschaft. München, Beck.

BECKER P. (2000), Unterwegs in der Landschaft - Wandern, Radfahren und Reiten. In: BECKER Ch., Јов H. (Hrsg.), Nationalatlas der Bundesrepublik Deutschland - Freizeit und Tourismus, S. 88-91. Heidelberg - Berlin, Spektrum Akad. Verlag. 
Beedie P., Hudson S. (2003), Emergence of Mountain-Based Adventure Tourism. In: Annals of Tourism Research, 30, 3, S. 625-643.

Berthelot L. (2006), An assessment of Via Alpina in France. Grenoble, unveröff. Manuskript. Bfin (Bundesamt Für Naturschutz), DTV (Deutscher Tourismusverband) (Hrsg.) (2005), Natur - Erlebnis - Angebote. Entwicklung und Vermarktung. Leitfaden. Bonn.

Borsdorf A. (2006), Via Alpina - Sustainability Evaluation. Innsbruck, unveröff. Manuskript. BRÄMER R. (2003), Natur tut uns gut. Warum wir uns beim Wandern so wohlfühlen. Vorlage für den Beitrag „Zurück zur Natur? - Die Wald- und Wiesen-Therapie“. In: Psychologie heute, 4, S. 20-28. - http://www.staff.uni-marburg.de/ braemer/natgut.htm (Abrufdatum: 5.7.2006)

BRÄMER R. (2005), Qualitätsoffensive Wandern. Auch in den Bergen? Würzburg, Manuskript zur Wegereferententagung des DAV am 30. April 2005 in Würzburg.

BrÄMER R. (2008), Die Wanderwelle. Neues aus der Wanderforschung. In: Wandermagazin, 143 , S. 8-9.

BROcKHAUs (2007), Brockhaus-Suche: wandern.- http://www.brockhaus-suche.de (Abrufdatum: 23.8.2007).

BRUnNer D. (2004), Wellness-Wanderer. In: Frankfurter Allgemeine Zeitung vom 15.7.2004, Reisebeilage, S. 1.

ButLer R.W. (1980), The Concept of a Tourist Area Cycle of Evolution: Implications for Management of Resources. In: Canadian Geographer, 24, 1, S. 5-12.

C-B-R Freizeit und Reisen (2005), Wandergipfel im Rahmen der C-B-R 2005 vom 19. bis 23. Februar 2005, Tourismusmesse.

Centouris (2008), Image- und Marktanalyse Ostbayern. Passau.

Clivaz Ch., Tornay V. (2006), L'évaluation de Via Alpina en Suisse. Sierre, Genf, unveröff. Manuskript.

Collins J.A., FAuser B.C.J.M. (2005), Balancing the strengths of systematic and narrative reviews. In: Human Reproduction Update, 11, 2, S. 103-104.

DGF (Deutsche Gesellschaft für Freizeit) (1999), Freizeit in Deutschland. Freizeittrends 2000plus. Erkrath.

Deutscher W AnderVerband (2004), Qualitätszeichen für Wanderwege in Deutschland . - http:// www.wanderverband.de/Pressel (Abrufdatum: 13.10.2004).

Deutscher Wanderverband (2006), Wanderbares Deutschland. - http://www.wanderbaresdeutschland.de (Abrufdatum: 12.7.2006).

Deutscher Wanderverband (2008), Wandern mit Qualität. Qualitätswege. - http://www. wanderbares-deutschland.de (Abrufdatum: 26.11.2008).

Doebeli H.P. (2003), Marktforschungsbericht „Wandern Schweiz“ (2003). Grundlagenforschung und Typologie des Wanderns der Schweizer Bevölkerung im Rahmen des nationalen Projektes „Swiss Alpine Walking“ zur nachhaltigen Förderung und Steigerung des Sommertourismus in der Schweiz (Revival des Produktes Wandern), durchgeführt für die Projektgruppe Swiss Alpine Walking, unterbreitet von Ernest Dichter SA. Zusammenfassung und Schlussfolgerungen. Zürich.

DVAG (Deutscher Verband für Angewandte Geographie) (2009), Fachtagung mit Exkursion „Megatrend Wandern - Chance zur Entwicklung ländlicher Regionen?“ am 12.-13. September 2009 in Gemünd, Tagung.

Educational Research Review (2008), An Author's Guide to Writing Articles and Reviews for Educational Research Review. - http://www.elsevier.com/framework_products/ promis_miscledurevguidetowriting.pdf (Abrufdatum: 12.11.2008).

Europäische Beobachtungsstelle Leader (Hrsg.) (2001), Wandertourismus im ländlichen Raum. Leitfaden für die Entwicklung und Durchführung von Wandertourismusprojekten (= Innovation im ländlichen Raum, 12). Brüssel. 
Focus (2005), Der Markt für Fitness und Wellness. Daten, Fakten, Trends. - http://www. dienstleister-info-ihk.de/branchen/Fitnesswirtschaft/Merkblaetter/05fitness.pdf (Abrufdatum: 4.7.2006).

FUR (Forschungsgemeinschaft Urlaub und Reisen) (2003), Reiseanalyse RA 2003. Kurzfassung. Hamburg - Kiel.

FUR (Forschungsgemeinschaft URlaub und Reisen) (2004), Die Urlaubsreisen der Deutschen. Kurzfassung Reiseanalyse 2004. Kiel.

FUR (Forschungsgemeinschaft URLaub und Reisen) (2005), Die Urlaubsreisen der Deutschen. Kurzfassung Reiseanalyse 2005. Köln.

FUR (Forschungsgemeinschaft URLAub Und Reisen) (2006), Die Urlaubsreisen der Deutschen. Kurzfassung der Reiseanalyse 2006. Kiel.

FUR (Forschungsgemeinschaft Urlaub und Reisen) (2007), Die Urlaubsreisen der Deutschen. Kurzfassung der Reiseanalyse 2007. Kiel.

FUR (Forschungsgemeinschaft URlaub und Reisen) (2008), Die Urlaubsreisen der Deutschen. Kurzfassung der Reiseanalyse 2008. Kiel.

Gerbert F. (2005), Die neue Wanderlust. In: Focus 2005, 20 vom 14.5.2005, S. 116-130.

Gerbert F. (2007), Qualitäts-, Prädikats- oder Premiumwege. Der Weg ist das Ziel - aber welcher? - http://www.focus.de/reisen/urlaubstipps/gerbert-wandert/qualitaets-praedikatsoder-premiumwege_aid_131209.html (Abrufdatum: 23.9.2008).

HaAs Ch. (2008), Wandertypologie. Sportler oder Entdecker? - http://www.focus.de/reisen/ reise-fuehrer/oesterreich/wandern/tid-11428/wandertypologie-sportler-oder-entdecker_ aid_324029.html (Abrufdatum: 23.9.2008).

Harrer B., Zeiner M., Maschke J., Scherr S. (1995), Tagesreisen der Deutschen. Struktur des Tagesausflugs- und Tagesgeschäftsreiseverkehrs in der Bundesrepublik Deutschland (= Schriftenreihe des dwif, 46). München.

HaUcK D. (1996), Trekkingtourismus in Nepal. Kulturgeographische Auswirkungen entlang der Trekkingrouten im vergleichenden Überblick (= Eichstätter Geogr. Arbeiten, 8). München.

Heidemann L. (2004), Wege ohne Ende - Wohin geht die „Wegemacherei“? In: Wege und Ziele. Weitwandern in Europa. Zeitschrift des Vereins Netzwerk Weitwandern e.V., 15, S. 4-9.

IFD (2007), AWA 2007. Freizeitbeschäftigungen. Auszug zur Verfügung gestellt vom Archiv des Instituts für Demoskopie, Allensbacher Werbeträgeranalyse per E-mail vom 8.12.2008.

IfD (Institut Für Demoskopie) (2007a), AWA 2007. Allensbacher Marktanalyse Werbeträgeranalyse. Codebuch. Allensbach.

IfD (Institut FÜr Demoskopie) (2008), Freizeitbeschäftigung Wandern 1986, 1990, 1995, 2000. Werte zur Verfügung gestellt vom Archiv des Instituts für Demoskopie, Allensbacher Werbeträgeranalyse per E-mail vom 8.12.2008.

IfD (Institut Für Demoskopie) (2008a), Untersuchungsdaten zur AWA 2008. - http://www. awa-online.de/pdf/udaten_08.pdf (Abrufdatum: 19.11.2008).

INVENT (VERBUNDPARTNER INVENT) (2005), INVENT Tourismus. Traumziel Nachhaltigkeit. Berlin.

ITB (2006), Podiumsdiskussion „Kreativ und gut zu Fuß - Wandern in Deutschland“ im Rahmen der ITB 2006 vom 10. bis 12. März 2006 in Berlin, Tagung im Rahmen der Tourismusmesse.

ITB (2008), ITB Berlin. 5.-9. März 2008. - http://www1.messe-berlin.de/vip8_1/website/Internet/Internet/www.itb-berlin/deutsch/Events/Events/PDFKatPressekonferenzen/index. jsp (Abrufdatum: 26.11.2008).

Јов Н. (2005), Die Alpen als Destination - Eine Analyse in vier Dimensionen. In: Mitt. d. Österr. Geogr. Ges., 147, S. 113-138.

Kundert R. (2006), Ein Volk macht mobil. In: Berge, 3, S. 34-39. 
LAND Vorarlberg (Hrsg.) (2004), Vorarlberger Wanderwege. - http://www.vorarlberg.at/wanderwege (Abrufdatum: 12.10.2004).

Leder S. (2003), Wandertourismus. In: Becker Ch., Hopfinger H., Steinecke A. (Hrsg.), Geographie der Freizeit und des Tourismus, S. 320-330. München - Wien.

LifeLine (2007), Ausdauersport. Wandern liegt wieder im Trend. - http://www.lifeline.de/ llspecial/fitness_sport/ausdauersport/content-128161.html (Abrufdatum: 23.9.2008).

MAnson J.E. et al. (1999), A prospective study of walking as compared with vigorous exercise in the prevention of coronary heart disease in women. In: The New England Journal of Medicine, August 26, S. 650-658.

Margreiter J. (2005), Auf neuen Wegen. In: Tirol Werbung (Hrsg.), Saison - Tourismusmagazin, 57,2 , S. 5.

Maschie J. (2005), Tagesreisen der Deutschen (= Schriftenreihe des dwif, 50). München.

Maschke J. (2007), Tagesreisen der Deutschen. Teil 3 (= Schriftenreihe des dwif, 52). München.

Messe Düsseldorf (2006), Presseabschlussmitteilung 3. Wander- und Trekkingmesse TourNatur Düsseldorf: Wandern ist auch bei Jüngeren zunehmend beliebt. - http://www.messeduesseldorf.deltournatur/de/index.html (Abrufdatum: 19.6.2006).

Messe DüsSELDORF (2007), Pressemeldungen. Anhaltender Wanderboom beschert TourNatur stetiges Wachstum. - http://www.tournatur.com/cipp/md_tournatur/custom/pub/content,lang,1/ oid,379/ticket,g_u_e_s_t/local_lang,1 (Abrufdatum: 26.11.2008).

Messe Düsseldorf (2008), Pressemeldungen. Abschluss-Pressemitteilung: TourNatur in der Wanderszene fest etabliert. - http://www.tournatur.com/cipp/md_tournatur/custom/pub/ content,lang,1/oid,458/ticket,g_u_e_s_t (Abrufdatum: 26.11.2008).

Messe München GmbH (2009), Suchergebnisse Neue Messe München. C-B-R Freizeit und Reisen. - http://www.messe-muenchen.de/index.php?cms-action:navigate=id:166 29277/lng:deltag: :online \&pagepart $=$ vdblist (Abrufdatum: 3.8.2009).

Messe Stuttgart (2008), ...und noch mehr Sonderurlaub! - http://www.messe-stuttgart.de/cms/ cmt-infos-besucher-schwerpunkte00.0.html (Abrufdatum: 3.8.2009).

Messe Stuttgart (2009), Das Besondere erleben. - http://cms.messe-stuttgart.de/cms $/ \mathrm{cmt}$ infos-besucher-schwerpunkte0000.0.html (Abrufdatum: 3.8.2009).

Neumeyer E., Dicks U. (2004), Wandern - ein attraktiver Baustein für nachhaltigen Tourismus. In: Brittner-Widmann A., Quack H.-D., Wachowiak H. (Hrsg.), Von Erholungsräumen zu Tourismusdestinationen: Facetten der Fremdenverkehrsgeographie (= Trierer Geogr. Studien, 27), S. 281-294.

NiederösterReich Information (2005), Übers weite Land. Niederösterreich - Reisen und Genießen 2005,1 . Wien.

Niederösterreich Werbung GmbH (2009), Niederösterreich neu erwandern. Wien.

N-TV (2006), Sportliche Singles erfolgreicher. Meldung vom 13.7.2006. - http://www.n-tv. de/688725.html (Abrufdatum: 19.7.2006).

NZZ (Neue Zürcher Zeitung) (2005), Wandern - Ein Klassiker boomt. Start eines Pilotprojekts für Qualitätssiegel in Bayern. In: Neue Zürcher Zeitung vom 21.4.2005, S. 55.

ÖsterReich Werbung (2009), Österreich. Von Natur aus wandern. - http://www.austria.info/del wandern (Abrufdatum: 3.8.2009).

Petermann Ch. (2008), Alles für die Walz. In: Migros-Magazin, 39, S. 33.

Pillkahn U. (2007), Trends und Szenarien als Werkzeuge der Strategieentwicklung. Erlangen.

Prantl D. (2007), Abkehr vom Gipfel. Die neuerliche Wanderlust äußert sich vor allem in ungewöhnlichen Varianten und einer immer anspruchsvolleren Kundschaft. In: Süddeutsche Zeitung vom 19.4.2007, S. 38.

PröBstl U. (2004), Planen für die Zukunft - Trends und ihre Auswirkungen auf die Erholungsplanung. In: Ländlicher Raum, 4, S. 18-24. 
Rauchfleisch U. (1994), Testpsychologie. Eine Einführung in die Psychodiagnostik. Göttingen - Zürich, Vandenhoeck \& Ruprecht.

Reisner U. (2004), Suche nach dem Klon. In: Tirol Werbung (Hrsg.), Saison - Tourismusmagazin, 56, 6, S. 12-19.

Schnöll F. (2005), Pfad der Erkenntnis. In: Tirol Werbung (Hrsg.), Saison - Tourismusmagazin, 57, 5, S. 28-29.

SEIFERT-RösING I. (2006), „Wandern ist in“ - von Trends und Thesen im Wandertourismus. In: Wanderzeit. Magazin des Deutschen Wanderverbandes, 5, 2, S. 4-5.

Seifert-Rösing I. (2007), Fitness: Durch Wandern gesund in den Herbst. - http://www.medizinaspekte.de/07/11/wellness_kuren/herbstwandern.html (Abrufdatum: 16.6.2010).

Spiess H. et al. (2008), Local Recreational Areas: Accounting for Peoples' Needs in the Development and Selection of Planning Instruments. In: Siegrist D. et al. (Hrsg.), Visitor Management in Nature-based Tourism. Strategies and Success Factors for Recreational and Protected Areas (= Series of the Institute for Landscape and Open Space), S. 107-118. Rapperswil, Switzerland.

Statista (2008), Was machen Sie in Ihrer Freizeit zumindest gelegentlich? - http://de.statista. org/statistik/diagramm/studie/88021/umfang/zumindest-gelegentlich-ausgeuebtefreizeitaktivitaeten/ (Abrufdatum: 19.11.2008).

Statista (2008a), Was machen Sie mehrmals im Monat in Ihrer Freizeit? -http://de.statista.org/ statistik/diagramm/studie/88020/umfrage/mehrmals-pro-monat-ausgeubte-freizeitaktivitaeten/ (Abrufdatum: 19.11.2008).

Statista (2008b), Wandern Sie in Ihrer Freizeit? - http://de.statista.org/statistik/diagramm/ studie/30398/umfrage/wandern-in-der-freizeit/ (Abrufdatum: 18.11.2008).

StATISTA (2008c), Welche Sportart(en) betreiben Sie? - http://de statista.org/statistik/diagramm/ studie/81147/umfragelausgeuebte-sportarten/ (Abrufdatum: 18.11.2008).

Statista (2008d), Was für Urlaubsreisen haben Sie in den letzten 12 Monaten gemacht? http://de.statista.org/statistik/diagramm/studie/87866/umfrage/art-der-urlaubsreisen/ (Abrufdatum: 18.11.2008).

Statista (2008e), Haben Sie in den letzten zwölf Monaten einen Wanderurlaub gemacht? - http:// de.statista.org/statistik/diagramm/studie/31455/umfrage/wanderurlaub-in-den-letzten12-monaten/ (Abrufdatum: 18.11.2008).

Statista (2008f), Planen Sie, in den kommenden 12 Monaten einen Wanderurlaub zu machen? - http://de.statista.org/statistik/diagramm/studie/31474/umfrage/wanderurlaub-innaechsten-12-monaten-geplant/ (Abrufdatum: 18.11.2008).

Statista (2008g), Haben Sie in den letzten zwölf Monaten einen Trekkingurlaub gemacht? - http://de.statista.org/statistik/diagramm/studie/31456/umfrage/trekkingurlaub-inden-letzten-12-monaten (Abrufdatum: 18.11.2008).

Statista (2008h), Planen Sie, in den kommenden 12 Monaten einen Trekkingurlaub zu machen? - http://de.statista.org/statistik/diagramm/studie/31475/umfrage/trekkingurlaub-innaechsten-12-monaten-geplant/ (Abrufdatum: 18.11.2008).

Statista (2009), Haben Sie in den letzten 12 Monaten einen Bade- oder Sonnenurlaub gemacht? - http://de.statista.org/statistik/diagramm/studie/31444/umfrage/bade--oder-sonnenurlaub-in-den-letzten-12-monaten/\#info (Abrufdatum: 31.7.2009).

STENGER M. (1998), Repräsentativerhebungen im Tourismus. Ein methodischer und inhaltlicher Vergleich (= Trierer Tourismus Bibliographien, 45).

Stephan J. (2007), Wandern ist der neue Trend. Auf den Spuren von Hape Kerkeling - Nur Vereine klagen. In: Erlanger Nachrichten vom 7.5.2007, S. 17. 
Stiftung Für Zukunftsfragen - eine Initiative von British American Tobacco (2008), Daten zur Freizeitforschung. Freizeit-Monitor 2007. Repräsentativbefragungen in Deutschland. Hamburg.

StFT (STUdienkreis Für Tourismus) (1991), Urlaubsreisen 1990. Kurzfassung der Reiseanalyse 1990. Starnberg.

StFT (STUdienkreis Für Tourismus) (1992), Urlaubsreisen 1991. Kurzfassung der Reiseanalyse 1991. Starnberg.

SZ (SüdDEutsche Zeitung) (2003), „Genug von Hightech-Hektik und Hyperstress”. Soziologe Rainer Brämer über das Wander-Bedürfnis einer neuen Generation und die perfekte Ausflugs-Dramaturgie. In: Süddeutsche Zeitung vom 5.9.2003, S. 18.

Thiene M., Scarpa R. (2008), Hiking in the Alps: exploring substitution patterns of hiking destinations. In: Tourism Economics, 14, 2, S. 263-282.

Thomas-Morus-Akademie (2005), Studienkonferenz „Trendsport Wandern? Fitness, Naturerlebnis, Sinnsuche..." am 18. Januar 2005 in Bergisch Gladbach, Tagung.

TIP DER Woche (2004), Tun Sie's doch auch mal wieder! Wandern ist cool. Keine Sportart ist so vielfältig - und keine so beliebt. In: Tip der Woche (= Kundenzeitung der KauflandGruppe) vom 18.10.2004, o. S.

Tirol InFo (2005), Du wirst Dich wandern. Tirol, Innsbruck.

Verband Deutscher Sportfachhandel (2008), 24.01.2008: In Europa blieb der Umsatz des Sportfachhandels 2007 mit rund 37 Milliarden Euro stabil - In Deutschland sank er von 7,1 Milliarden auf 6,9 Milliarden. - http://www.vds-sportfachhandel.de/index. php?id=2\&sub=3\&DS=123 (Abrufdatum: 8.12.2008).

Verband Deutscher Sportfachhandel (2008a), 20.11.2008: Aktuelle Verkaufszahlen: Outdoorhosen (KW 45/2008). - http://www.vds-sportfachhandel.de/index.php?id=2\&sub= $3 \& D S=169$ (Abrufdatum: 8.12.2008).

Via Alpina (2008), Via Alpina. Discover the Alps! Funding. - http://www.via-alpina.org/Default. aspx? pageid=page78 (Abrufdatum: 10.11.2008).

Vogt L. (2008), Regionalentwicklung peripherer Räume mit Tourismus? Eine akteur- und handlungsorientierte Untersuchung am Beispiel des Trekkingprojekts Grande Traversata delle Alpi (= Erlanger Geogr. Arbeiten, Sonderbde., 38).

Vogt L. (2009), Wandern und Trekking als Freizeitaktivität und Marktsegment im Naturtourismus. Ein Überblick über den Stand der Kenntnisse und ein Ausblick auf landschaftsplanerische Konsequenzen. In: Naturschutz und Landschaftsplanung, 41, 8, S. 229-236.

Vogt L. et al. (2006), Projektbericht - Evaluation der Via Alpina in Deutschland 2005. Erlangen (= unveröff. Manuskript)

W AHRig-Burfeind R. (Hrsg.) (2002), Wahrig - Deutsches Wörterbuch. Gütersloh, München.

W ANDERMAgazin (2006), Boom der Wandermessen. Spezialmessen bedienen Mehrheitsinteressen. In: Wandermagazin Februar/März, S. 36-37.

Wenzel E., Rauch Ch. (2008), Best Ager - Der Silberne Markt. - http://portal.wko.at/wk/dok_detail_file.wk?AngID=1\&DocID=887928\&ConID=331499 (Abrufdatum: 26.11.2008).

Werner S. (2005), Trend Seniorenwandern. Gipfelstürmer mit 70. - http://www.spiegel.del reise/aktuell/0,1518,druck-368284,00.html (Abrufdatum: 10.11.2008).

WTO (World Tourism Organization) (2007), 2007: Full Steam ahead for International Tourism. Latest UNWTO World Tourism Barometer. - http://www.unwto.org/media/news/ en/press_det.php?id=1501\&idioma $=E$ (Abrufdatum: 11.11.2008). 